Pacific

Journal of

Mathematics

NORMAL FORMS FOR CR SINGULAR CODIMENSION-TWO LEVI-FLAT SUBMANIFOLDS

XIANGHONG GONG AND JIŘí LEBL 


\title{
NORMAL FORMS FOR CR SINGULAR CODIMENSION-TWO LEVI-FLAT SUBMANIFOLDS
}

\author{
XiANGHONG GONG AND JiŘí LEBL
}

\begin{abstract}
Real-analytic Levi-flat codimension-two CR singular submanifolds are a natural generalization to $\mathbb{C}^{m}, m>2$, of Bishop surfaces in $\mathbb{C}^{2}$. Such submanifolds, for example, arise as zero sets of mixed-holomorphic equations with one variable antiholomorphic. We classify the codimension-two Leviflat CR singular quadrics, and we notice that new types of submanifolds arise in dimension three or higher. In fact, the nondegenerate submanifolds, i.e., higher order perturbations of $z_{m}=\bar{z}_{1} z_{2}+\bar{z}_{1}^{2}$, have no analogue in dimension two. We prove that the Levi foliation extends through the singularity in the real-analytic nondegenerate case. Furthermore, we prove that the quadric is a (convergent) normal form for a natural large class of such submanifolds, and we compute its automorphism group. In general, we find a formal normal form in $\mathbb{C}^{3}$ in the nondegenerate case that shows infinitely many formal invariants.
\end{abstract}

1. Introduction

116

2. Invariants of codimension-two CR singular submanifolds $\quad 120$

3. Levi-flat quadrics 122

4. Quadratic Levi-flat submanifolds and their Segre varieties 130

5. The CR singularity of Levi-flat quadrics 131

6. Levi foliations and images of generic Levi-flats 132

7. Extending the Levi foliation of type C.x submanifolds 135

8. CR singular set of type C.x submanifolds 137

9. Mixed-holomorphic submanifolds 138

10. Formal normal form for certain type C.1 submanifolds I 140

11. Convergence of normalization for certain type C.1 submanifolds 142

12. Automorphism group of the C.1 quadric 144

13. Normal form for certain type C.1 submanifolds II 148

14. Normal form for general type C.1 submanifolds 151

15. Instability of Bishop-like submanifolds 161

$\begin{array}{ll}\text { References } & 163\end{array}$

Lebl was in part supported by NSF grant DMS 0900885 and DMS 1362337.

MSC2010: primary 32V40; secondary 53C12, 32S05.

Keywords: Normal form, Levi-flat, CR singular, codimension two, Bishop surface, mixed-holomorphic equations. 


\section{Introduction}

Let $M \subset \mathbb{C}^{n+1}$ be a real submanifold. A fundamental question in CR geometry is to classify $M$ at a point up to local biholomorphic transformations. One approach is to find a normal form for $M$.

A real-analytic hypersurface $M \subset \mathbb{C}^{n+1}$ is Levi-flat if the Levi form vanishes identically. Roughly speaking, a Levi-flat submanifold is a family of complex submanifolds. Intuitively, a Levi-flat submanifold is as close to a complex submanifold as possible. In the real-analytic smooth hypersurface case, it is well known that $M$ can locally be transformed into the real hyperplane given by

$$
\operatorname{Im} z_{1}=0 .
$$

We therefore focus on higher codimension case, in particular on codimension two. A codimension-two submanifold is again given by a single equation, but in this case a complex valued equation. A new phenomenon that appears in codimension two is that $M$ may no longer be a CR submanifold. Let $T_{p}^{c} M \subset T_{p} M$ be the largest subspace with $J T_{p}^{c} M=T_{p}^{c} M$, where $J$ is the complex structure on $\mathbb{C}^{n+1}$. A submanifold is CR if $\operatorname{dim} T_{p}^{c} M$ is constant.

Real submanifolds of dimension $n+1$ in $\mathbb{C}^{n+1}$ with a nondegenerate complex tangent point has been studied extensively after the fundamental work of E. Bishop [1965]. In $\mathbb{C}^{2}$, he studied the submanifolds

$$
w=z \bar{z}+\gamma\left(z^{2}+\bar{z}^{2}\right)+O(3),
$$

where $\gamma \in[0, \infty]$ is called the Bishop invariant, with $\gamma=\infty$ interpreted as $w=$ $z^{2}+\bar{z}^{2}+O(3)$. One of Bishop's motivations was to study the hull of holomorphy of the real submanifolds by attaching analytic discs. His work on the family of attached analytic discs has been refined by Kenig and Webster [1982; 1984], Huang and Krantz [1995], and Huang [1998]. The normal form theory for real submanifolds for Bishop surfaces or submanifolds was established by Moser and Webster [1983]; see also Moser [1985], Gong [1994a; 1994b; 2004], Huang and Yin [2009a], and Coffman [2010]. We mention that the Moser-Webster normal form does not deal with the case of vanishing Bishop invariant.

The formal normal form and its application to holomorphic classification for surfaces with vanishing Bishop invariant were achieved by Huang and Yin [2009a] by a completely different method. Real submanifolds with complex tangents have been studied in other situations. See, for example, [Lebl et al. 2014], where CR singular submanifolds that are images of CR manifolds were studied. Normal forms for the quadratic part of general codimension-two CR singular submanifolds in $\mathbb{C}^{3}$ was completely solved by Coffman [2009]. Huang and Yin [2009b] studied the normal form for codimension-two CR singular submanifolds of the form $w=$ 
$|z|^{2}+O(3)$. Dolbeault, Tomassini and Zaitsev [2005; 2011] and Huang and Yin [2012] studied CR singular submanifolds of codimension-two that are boundaries of Levi-flat hypersurfaces. Burcea [2013] constructed the formal normal form for codimension-two CR singular submanifolds approximating a sphere. Coffman [2006] found an algebraic normal form for nondegenerate CR singular manifolds in high codimension and one-dimensional complex tangent.

To motivate our work, we observe that in Bishop's work, the real submanifolds are Levi-flat away from their CR singular sets. Our purpose is to understand such submanifolds in higher dimensional case with codimension being exactly two. Notice that the latter is the smallest codimension for CR singularity to be present in (smooth) submanifolds. Regarding CR singular Levi-flat real codimension-two submanifolds on $\mathbb{C}^{n+1}$ as a natural generalization of Bishop surfaces to $\mathbb{C}^{n+1}$, we wish to find their normal forms. For singular Levi-flat hypersurfaces and related work on foliations with singularity, see [Bedford 1977; Burns and Gong 1999; Brunella 2007; Cerveau and Lins Neto 2011; Lebl 2013; Fernández-Pérez 2013].

Our techniques revolve around the study of the Levi map (the generalization of the Levi form to higher codimension submanifolds) of codimension-two submanifolds. Extending the CR structure through the singular point via Nash blowup and then extending the Levi map to this blowup has been studied previously by Garrity [2000].

A CR submanifold is Levi-flat if the Levi map vanishes identically. Locally, all CR real-analytic Levi-flat submanifolds of real codimension two can be, after holomorphic change of coordinates, written as

$$
\operatorname{Im} z_{1}=0, \quad \operatorname{Im} z_{2}=0 .
$$

If a submanifold $M$ is CR singular, denote by $M_{\mathrm{CR}}$ the set of points where $M$ is CR. We say $M$ is Levi-flat if $M_{\mathrm{CR}}$ is Levi-flat in the usual sense. A Levi-flat CR singular submanifold has no local biholomorphic invariants at the CR points, just as in the case of Bishop surfaces.

A real, real-analytic codimension-two submanifold that is $\mathrm{CR}$ singular at the origin can be written in coordinates $(z, w) \in \mathbb{C}^{n} \times \mathbb{C}=\mathbb{C}^{n+1}$ as

$$
w=\rho(z, \bar{z})
$$

for $\rho$ that is $O(2)$. We will be concerned with submanifolds where the quadratic part in $\rho$ is nonzero in any holomorphic coordinates. We say that such submanifolds have a nondegenerate complex tangent. For example, the Bishop surfaces in $\mathbb{C}^{2}$ are precisely the CR singular submanifolds with nondegenerate complex tangent.

First, let us classify the quadratic parts of CR singular Levi-flats, and in the process completely classify the CR singular Levi-flat quadrics, that is, those where $\rho$ is a quadratic. 
Theorem 1.1. Suppose that $M \subset \mathbb{C}^{n+1}, n \geq 2$, is a germ of a real-analytic real codimension-two submanifold, CR singular at the origin, written in coordinates $(z, w) \in \mathbb{C}^{n} \times \mathbb{C}$ as

$$
w=A(z, \bar{z})+B(\bar{z}, \bar{z})+O(3),
$$

for quadratic $A$ and $B$, where $A+B \not \equiv 0$ (nondegenerate complex tangent). Suppose that $M$ is Levi-flat (that is, $M_{\mathrm{CR}}$ is Levi-flat).

(i) If $M$ is a quadric, then $M$ is locally biholomorphically equivalent to one and exactly one of the following:

$$
\begin{aligned}
& w=\bar{z}_{1}^{2}, \\
& w=\bar{z}_{1}^{2}+\bar{z}_{2}^{2},
\end{aligned}
$$

$\vdots$

$$
\begin{aligned}
& w=\bar{z}_{1}^{2}+\bar{z}_{2}^{2}+\cdots+\bar{z}_{n}^{2}, \\
& w=\left|z_{1}\right|^{2}+\gamma \bar{z}_{1}^{2}, \quad \gamma \geq 0, \\
& w=\bar{z}_{1} z_{2}, \\
& w=\bar{z}_{1} z_{2}+\bar{z}_{1}^{2} .
\end{aligned}
$$

(ii) If $M$ is real-analytic, then the quadric

$$
w=A(z, \bar{z})+B(z, \bar{z})
$$

is Levi-flat, and can be put via a biholomorphic transformation into exactly one of the forms above.

By part (ii), the quadratic part in (5) is an invariant of $M$ at a point. We say the type of $M$ at the origin is A.x, B. $\gamma$, or C.x depending on the type of the quadratic form. Following Bishop, we call types B. $\gamma$ and A.1 Bishop-like, and we could think of $\gamma=\infty$ as A.1.

By type being stable we mean that the type does not change at all complex tangents in a neighborhood of the origin under any small (or higher order) perturbations that stay within the class of Levi-flat CR singular submanifolds. As a consequence of Theorem 1.1 and because the rank is lower semicontinuous, we get that the only types that are stable are A. $n$ and C.1, although A. $n$ are degenerate because the form $A(z, \bar{z})$ is identically zero. See also Proposition 15.1.

The quadrics A. $k$ for $k \geq 2$ do not possess a nonsingular foliation extending the Levi foliation of $M_{\mathrm{CR}}$ through the origin. In fact, there is a singular complex subvariety of dimension one through the origin contained in $M$. See Section 6 .

In the sequel, when we wish to refer to the quadric of certain type we will use the notation $M_{C .1}$ to denote the quadric of type C.1. 
The quadratic form $A(z, \bar{z})$ carries the "Levi map" of the submanifold. Type C.1 is the unique quadric that is stable and has nonzero $A$. Having nonzero $A$ is also stable in a neighborhood of the origin under any small (or higher order) perturbations. Therefore, we say a type is nondegenerate if it is C.1 and we focus mostly on such submanifolds. First, we show that submanifolds of type C.x possess a nonsingular real-analytic foliation that extends the Levi foliation, due to the form $A(z, \bar{z})$ :

Theorem 1.2. Suppose that $M \subset \mathbb{C}^{n+1}, n \geq 2$, is a real-analytic Levi-flat $C R$ singular submanifold of type $C .1$ or C.0, that is, $M$ is given by

$$
w=\bar{z}_{1} z_{2}+\bar{z}_{1}^{2}+O(3) \text { or } \quad w=\bar{z}_{1} z_{2}+O(3) .
$$

Then there exists a nonsingular real-analytic foliation defined on $M$ that extends the Levi foliation on $M_{\mathrm{CR}}$; and consequently, there exists a CR real-analytic mapping $F: U \subset \mathbb{R}^{2} \times \mathbb{C}^{n-1} \rightarrow \mathbb{C}^{n+1}$ such that $F$ is a diffeomorphism onto $F(U)=M \cap U^{\prime}$, for some neighborhood $U^{\prime}$ of zero.

Here the $\mathrm{CR}$ structure on $\mathbb{R}^{2} \times \mathbb{C}^{n-1}$ is induced from $\mathbb{C}^{2} \times \mathbb{C}^{n-1}$. As a corollary of this theorem we obtain in Section 8 using the results of [Lebl et al. 2014] that the CR singular set of any type C.1 submanifold is a Levi-flat submanifold of dimension $2 n-2$ and $\mathrm{CR}$ dimension $n-2$.

The Levi foliation on a type C.x submanifold cannot extend to a whole neighborhood of $M$ as a nonsingular holomorphic foliation. If it did, we could flatten the foliation and $M$ would be a Cartesian product, in particular Bishop-like. Thus, the study of normal form theory for the special case when the foliation extends to a neighborhood is reduced to the case of Bishop surfaces, which have been studied extensively.

A codimension-two submanifold in $\mathbb{C}^{m}$ can arise from

$$
f\left(\bar{z}^{\prime}, z^{\prime \prime}\right)=0
$$

for a suitable holomorphic function $f$ in $m$ variables. The zero set admits two holomorphic foliations. We are interested in the case where one of foliations has leaves of maximum dimension $m-2$, while the other has leaves of minimum dimension zero. Therefore, we will assume that $z^{\prime}=z_{1}$ and $z^{\prime \prime}=\left(z_{2}, \ldots, z_{m}\right)$. Functions holomorphic in some variables and anti-holomorphic in other variables, such as (8), are often called mixed-holomorphic or mixed-analytic, and come up often in complex geometry, the simplest example being the standard inner product. An interesting feature of the mixed-holomorphic setting is that the equation can be complexified into $\mathbb{C}^{m}$, so the sets share some of the properties of complex varieties. However, they have a different automorphism group if we wish to classify them under biholomorphic transformations. Such mixed-analytic sets are automatically real codimension two, are Levi-flat or complex, and may have CR singularities. We study their normal form in Section 9. See also Theorem 1.3 below. 
When a type C.1 CR singular submanifold has a defining equation that does not depend on $\bar{z}_{2}, \ldots, \bar{z}_{n}$ we prove that it is automatically Levi-flat, and it is equivalent to $M_{C .1}$.

Theorem 1.3. Let $M \subset \mathbb{C}^{n+1}, n \geq 2$, be a real-analytic submanifold given by

$$
w=\bar{z}_{1} z_{2}+\bar{z}_{1}^{2}+r\left(z_{1}, \bar{z}_{1}, z_{2}, z_{3}, \ldots, z_{n}\right),
$$

where $r$ is $O(3)$. Then $M$ is Levi-flat and at the origin and $M$ is locally biholomorphically equivalent to the quadric $M_{C .1}$ submanifold

$$
w=\bar{z}_{1} z_{2}+\bar{z}_{1}^{2} \text {. }
$$

The theorem is also true formally; given a formal submanifold of the form (9), it is formally equivalent to $M_{C .1}$.

A key idea in the proof of the convergence of the normalizing transformation is that the form $B(\bar{z}, \bar{z})=\bar{z}_{1}^{2}$ induces a natural mixed-holomorphic involution on quadric $M_{C .1}$. This involution also plays a key role in computing the automorphism group of the quadric in Theorem 12.4.

Finally, we also compute the automorphism group for the quadric $M_{C .1}$; see Theorem 12.4. In particular we show that the automorphism group is infinitedimensional.

Not every type C.1 Levi-flat submanifold is biholomorphically equivalent to the C.1 quadric. We will find a formal normal form for type C.1 Levi-flat submanifolds in $\mathbb{C}^{3}$ that shows infinitely many formal invariants. Let us give a simplified statement. For details, see Theorem 14.3.

Theorem 1.4. Let $M$ be a real-analytic Levi-flat type C.1 submanifold in $\mathbb{C}^{3}$. There exists a formal biholomorphic map transforming $M$ into the image of

$$
\widehat{\varphi}(z, \bar{z}, \xi)=(z+A(z, \xi, w) w \eta, \xi, w)
$$

with $\eta=\bar{z}+\frac{1}{2} \xi$ and $w=\bar{z} \xi+\bar{z}^{2}$. Here $A=0$, or A satisfies certain normalizing conditions.

When $A \neq 0$, the formal automorphism group preserving the normal form is finite or one-dimensional.

We do not know if the formal normal form above can be achieved by convergent transformations, even if $A=0$.

\section{Invariants of codimension-two CR singular submanifolds}

Before we impose the Levi-flat condition, let us find some invariants of codimensiontwo CR singular submanifolds in $\mathbb{C}^{n+1}$ with $\mathrm{CR}$ singularity at zero. Such a submanifold, locally near the origin, can be put into the form

$$
w=A(z, \bar{z})+B(\bar{z}, \bar{z})+O(3),
$$


where $(z, w) \in \mathbb{C}^{n} \times \mathbb{C}$ and $A$ and $B$ are quadratic forms. We think of $A$ and $B$ as matrices and $z$ a column vector and write the forms as $z^{*} A z$ and $z^{*} B \bar{z}$ respectively. The matrix $B$ is not unique. Hence we make $B$ symmetric to make the choice of the matrix $B$ canonical. The following proposition is not difficult and well known. Since the details are important and will be used later, let us prove:

Proposition 2.1. A biholomorphic transformation of (12) taking the origin to itself and preserving the form of (12) takes the matrices $(A, B)$ to

$$
\left(\lambda T^{*} A T, \lambda T^{*} B \bar{T}\right),
$$

for $T \in \mathrm{GL}_{n}(\mathbb{C})$ and $\lambda \in \mathbb{C}^{*}$. If $\left(F_{1}, \ldots, F_{n}, G\right)=(F, G)$ is the transformation, then the linear part of $G$ is $\lambda^{-1} w$ and the linear part of $F$ restricted to $z$ is $T z$.

Let us emphasize that $A$ is an arbitrary complex matrix and $B$ is symmetric, but not necessarily Hermitian.

Proof. Let $\left(F_{1}, \ldots, F_{n}, G\right)=(F, G)$ be a change of coordinates taking

$$
w=\widetilde{A}(z, \bar{z})+\widetilde{B}(\bar{z}, \bar{z})+O(3)=\rho(z, \bar{z})
$$

to

$$
w=A(z, \bar{z})+B(\bar{z}, \bar{z})+O(3) .
$$

Then

$$
\begin{aligned}
& G(z, \rho(z, \bar{z})) \\
& =A(F(z, \rho(z, \bar{z})), \bar{F}(\bar{z}, \bar{\rho}(\bar{z}, z)))+B(\bar{F}(\bar{z}, \bar{\rho}(\bar{z}, z)), \bar{F}(\bar{z}, \bar{\rho}(\bar{z}, z)))+O(3)
\end{aligned}
$$

is true for all $z$. The right hand side has no linear terms, so the linear terms in $G$ do not depend on $z$. That is, $G=\lambda^{-1} w+O(2)$, where $\lambda$ is a nonzero scalar and the negative power is for convenience.

Let $T=\left[T_{1}, T_{2}\right]$ denote the matrix representing the linear terms of $F$. Here $T_{1}$ is an $n \times n$ matrix and $T_{2}$ is $n \times 1$. Since the linear terms in $G$ do not depend on any $z_{j}, T_{1}$ is nonsingular. Then the quadratic terms in (16) are

$$
\lambda^{-1}(\tilde{A}(z, \bar{z})+\widetilde{B}(\bar{z}, \bar{z}))=z^{*} T_{1}^{*} A T_{1} z+z^{*} T_{1}^{*} B \bar{T}_{1} \bar{z} .
$$

In other words as matrices,

$$
\widetilde{A}=\lambda T_{1}^{*} A T_{1} \quad \text { and } \quad \widetilde{B}=\lambda T_{1}^{*} B \bar{T}_{1} .
$$

We will need to at times reduce to the three-dimensional case, and so we need: Lemma 2.2. Let $M \subset \mathbb{C}^{n+1}, n \geq 3$, be a real-analytic Levi-flat $C R$ singular submanifold of the form

$$
w=A(z, \bar{z})+B(\bar{z}, \bar{z})+O(3),
$$


where $A$ and $B$ are quadratic. Let $L$ be a nonsingular $(n-2) \times n$ matrix. If $A+B$ is not zero on the set $\{L z=0\}$, then the submanifold

$$
M_{L}=M \cap\{L z=0\}
$$

is a Levi-flat CR singular submanifold.

Proof. Clearly, if $M_{L}$ is not contained in the CR singularity of $M$, then $M_{L}$ is a Levi-flat CR singular submanifold. $M_{L^{\prime}}$ is not contained in the CR singularity of $M$ for a dense open subset of $(n-2) \times n$ matrices $L^{\prime}$. If $M_{L}$ is a subset of the CR singularity of $M$, pick a CR point $p$ of $M_{L}$ then pick a sequence $L_{n}$ approaching $L$ such that $M_{L_{n}}$ are not contained in the CR singularity of $M$. As $A+B$ is not zero on the set $\{L z=0\}$, then $M_{L}$ is not a complex submanifold, and therefore a CR singular submanifold. As the Levi form of $M_{L_{n}}$ vanishes at all CR points of $M_{L_{n}}$, the Levi form of $M_{L}$ vanishes at $p$, so $M_{L}$ is Levi-flat.

\section{Levi-flat quadrics}

Let us first focus on Levi-flat quadrics. We will prove later that the quadratic part of a Levi-flat submanifold is Levi-flat. Let $M$ be defined in $(z, w) \in \mathbb{C}^{n} \times \mathbb{C}$ by

$$
w=A(z, \bar{z})+B(\bar{z}, \bar{z}) .
$$

Being Levi-flat has several equivalent formulations. The main idea is that the $T^{(1,0)} M \times T^{(0,1)} M$ vector fields are completely integrable at CR points and we obtain a foliation of $M$ at CR points by complex submanifolds of complex dimension $n-1$. An equivalent notion is that the Levi map is identically zero; see [Baouendi et al. 1999]. The Levi map for a CR submanifold defined by two real equations $\rho_{1}=\rho_{2}=0$ (for $\rho_{1}$ and $\rho_{2}$ with linearly independent differentials) is the pair of Hermitian forms

$$
i \partial \bar{\partial} \rho_{1} \quad \text { and } \quad i \partial \bar{\partial} \rho_{2} \text {, }
$$

applied to $T^{(1,0)} M$ vectors. The full quadratic forms $i \partial \bar{\partial} \rho_{1}$ and $i \partial \bar{\partial} \rho_{2}$ of course depend on the defining equations themselves and are therefore extrinsic information. It is important to note that for the Levi map we restrict it to $T^{(1,0)} M$ vectors. We can define these two forms $i \partial \bar{\partial} \rho_{1}$ and $i \partial \bar{\partial} \rho_{2}$ even at a CR singular point $p \in M$.

These forms are the complex Hessian matrices of the defining equations. For our quadric $M$, they are the real and imaginary parts of the $(n+1) \times(n+1)$ complex matrix

$$
\tilde{A}=\left[\begin{array}{ll}
A & 0 \\
0 & 0
\end{array}\right],
$$

where the variables are ordered as $\left(z_{1}, \ldots, z_{n}, w\right)$. 
For $M$ to be Levi-flat, the quadratic form defined by $\widetilde{A}$ has to be zero when restricted to the $(n-1)$-dimensional space spanned by $T_{p}^{(1,0)} M$ for every $p \in M_{\mathrm{CR}}$. In other words, for every $p \in M_{\mathrm{CR}}$,

$$
v^{*} \widetilde{A} v=0 \text { for all } v \in T_{p}^{(1,0)} M .
$$

The space $T_{p}^{(1,0)} M$ is of dimension $n-1$, and furthermore, the vector $\partial / \partial w$ is not in $T_{p}^{(1,0)} M$. Therefore, $z^{*} A z=0$ for $z \in \mathbb{C}^{n}$ in a subspace of dimension $n-1$.

Before we proceed, let us note the following general fact about CR singular Levi-flat submanifolds:

Lemma 3.1. Suppose that $M \subset \mathbb{C}^{n+1}, n \geq 2$, is a Levi-flat connected real-analytic real codimension-two submanifold, $C R$ singular at the origin. Then there exists a germ of a complex analytic variety of complex dimension $n-1$ through the origin, contained in $M$.

Proof. Through each point of $M_{\mathrm{CR}}$ there exists a germ of a complex variety of complex dimension $n-1$ contained in $M$. The set of CR points is dense in $M$. Take a sequence $p_{k}$ of CR points converging to the origin and take complex varieties of dimension $n-1, W_{k} \subset M$ with $p_{k} \in W_{k}$. A theorem of Fornæss (see [Kohn 1979, Theorem 6.23] for a proof using the methods of Diederich and Fornæss [1978]) implies that there exists a variety through $W \subset M$ with $0 \in W$ and of complex dimension at least $n-1$.

Let us first concentrate on $n=2$, in which case $T^{(1,0)} M$ is one-dimensional at CR points. Write

$$
A=\left[\begin{array}{ll}
a_{11} & a_{12} \\
a_{21} & a_{22}
\end{array}\right], \quad B=\left[\begin{array}{ll}
b_{11} & b_{12} \\
b_{12} & b_{22}
\end{array}\right] .
$$

Note that $B$ is symmetric. A short computation shows that the vector field can be written as

$$
\alpha \frac{\partial}{\partial w}+\beta_{1} \frac{\partial}{\partial z_{1}}+\beta_{2} \frac{\partial}{\partial z_{2}}=\alpha \frac{\partial}{\partial w}+\beta \frac{\partial}{\partial z}
$$

where

$$
\begin{aligned}
\beta_{1} & =\bar{a}_{21} \bar{z}_{1}+\bar{a}_{22} \bar{z}_{2}+2 \bar{b}_{12} z_{1}+2 \bar{b}_{22} z_{2}, \\
\beta_{2} & =-\bar{a}_{11} \bar{z}_{1}-\bar{a}_{12} \bar{z}_{2}-2 \bar{b}_{11} z_{1}-2 \bar{b}_{12} z_{2}, \\
\alpha & =a_{11} \bar{z}_{1} \beta_{1}+a_{21} \bar{z}_{2} \beta_{1}+a_{12} \bar{z}_{1} \beta_{2}+a_{22} \bar{z}_{2} \beta_{2} .
\end{aligned}
$$

$M_{\mathrm{CR}}$ is dense in $M$, since the CR singular set is defined by $\beta_{1}=\beta_{2}=0$. Thus for $M$ to be Levi-flat we need to check that the following product is identically zero:

$$
\left[\begin{array}{ll}
\beta^{*} & \bar{\alpha}
\end{array}\right]\left[\begin{array}{cc}
A & 0 \\
0 & 0
\end{array}\right]\left[\begin{array}{l}
\beta \\
\alpha
\end{array}\right]=\beta^{*} A \beta .
$$


If $A$ is the zero matrix, then $M$ is automatically Levi-flat. We diagonalize $B$ via $T$ into a diagonal matrix with ones and zeros on the diagonal. We obtain (recall $n=2)$ the submanifolds

$$
w=\bar{z}_{1}^{2} \quad \text { or } \quad w=\bar{z}_{1}^{2}+\bar{z}_{2}^{2} .
$$

The first submanifold is of the form $M \times \mathbb{C}$, where $M \subset \mathbb{C}^{2}$ is a Bishop surface.

Let us from now on suppose that $A \neq 0$.

As $M$ is Levi-flat, then through each CR point $p=\left(z_{p}, w_{p}\right) \in M_{\mathrm{CR}}$ we have a complex submanifold of dimension one in $M$. It is well known that this submanifold is contained in the Segre variety (see also Section 4)

$$
w=A\left(z, \bar{z}_{p}\right)+B\left(\bar{z}_{p}, \bar{z}_{p}\right), \quad \bar{w}_{p}=\bar{A}\left(\bar{z}_{p}, z\right)+\bar{B}(z, z) .
$$

By Lemma 3.1 we obtain a complex variety $V \subset M$ of dimension one through the origin. Suppose without loss of generality that $V$ is irreducible. $V$ has to be contained in the Segre variety at the origin, in particular $w=0$ on $V$. Therefore, to simplify notation, let us consider $V$ to be subvariety of $\{w=0\}$. Denote by $\bar{V}$ the complex conjugate of $V$. Then as $V$ is irreducible, $V \times \bar{V}$ is also irreducible (the smooth part of $V$ is connected and so is the smooth part of $V \times \bar{V}$; see [Whitney 1972]). Hence, by complexifying, we have $A(z, \bar{\xi})+B(\bar{\xi}, \bar{\xi})=0$ for all $z \in V$ and $\xi \in V$.

If $B \neq 0$, then setting $z=0$, we have $B(\bar{\xi}, \bar{\xi})=0$ on $V$. As $B$ is homogeneous and $V$ is irreducible, $V$ is a one-dimensional complex line. If $B=0$, then $A(z, \bar{\zeta})=0$ for $z, \zeta \in V$ as mentioned above. We consider two cases. Suppose first that every $\sum_{j=1}^{2} a_{i j} \bar{\zeta}_{j}$ is identically zero for all $\zeta \in V$ and $i=1,2$. Then $V$ is contained in some complex line $\sum_{j=1}^{2} \bar{a}_{i j} \zeta_{j}=0$. Suppose now that $A\left(z, \bar{\zeta}_{*}\right)$ is not identically zero for some $\zeta_{*} \in V$. Then $V$ is contained in the complex line $A\left(z, \bar{\zeta}_{*}\right)=0$. This shows that $V$ is a complex line.

Thus as $A(z, \bar{z})+B(\bar{z}, \bar{z})$ is zero on a one-dimensional linear subspace, we make this subspace $\left\{z_{1}=0\right\}$ and so each monomial in $A(z, \bar{z})+B(\bar{z}, \bar{z})$ is divisible by either $z_{1}$ or $\bar{z}_{1}$. Therefore, $A$ and $B$ are matrices of the form

$$
\left[\begin{array}{ll}
* & * \\
* & 0
\end{array}\right]
$$

that is, $a_{22}=0$ and $b_{22}=0$.

To normalize the pair $(A, B)$, we apply arbitrary invertible transformations $(T, \lambda) \in \mathrm{GL}_{n}(\mathbb{C}) \times \mathbb{C}^{*}$ as

$$
(A, B) \mapsto\left(\lambda T^{*} A T, \lambda T^{*} B \bar{T}\right) .
$$


Recall that we are assuming that $A \neq 0$. If $a_{21}=0$ or $a_{12}=0$, then $A$ has rank one, and via a transformation $T$ of the form

$$
z_{1}^{\prime}=z_{1}, \quad z_{2}^{\prime}=z_{2}+c z_{1} \quad \text { or } \quad z_{2}^{\prime}=z_{1}, \quad z_{1}^{\prime}=z_{2}+c z_{1}
$$

and rescaling by nonzero $\lambda$, the matrix $A$ can be put in the form

$$
\left[\begin{array}{ll}
0 & 1 \\
0 & 0
\end{array}\right] \text { or }\left[\begin{array}{ll}
1 & 0 \\
0 & 0
\end{array}\right] \text {. }
$$

The transformation $T$ and $\lambda$ must also be applied to $B$ and this could possibly make $b_{22} \neq 0$. However, we will show that we actually have $b_{22}=0$. Thus $B=0$ on $z_{1}=0$ still holds true.

Let us first focus on

$$
A=\left[\begin{array}{ll}
1 & 0 \\
0 & 0
\end{array}\right]
$$

We apply the $T^{(1,0)}$ vector field computed above. Only $a_{11}$ is nonzero in $A$. Therefore $\beta^{*} A \beta$, which must be identically zero, is

$$
\begin{aligned}
0=\beta^{*} A \beta & =\bar{\beta}_{1} \beta_{1}=\overline{\left(2 \bar{b}_{12} z_{1}+2 \bar{b}_{22} z_{2}\right)}\left(2 \bar{b}_{12} z_{1}+2 \bar{b}_{22} z_{2}\right) \\
& =4\left(\left|b_{12}\right|^{2} z_{1} \bar{z}_{1}+\left|b_{22}\right|^{2} z_{2} \bar{z}_{2}+b_{12} \bar{b}_{22} \bar{z}_{1} z_{2}+\bar{b}_{12} b_{22} z_{1} \bar{z}_{2}\right) .
\end{aligned}
$$

This polynomial must be identically zero and hence all coefficients must be identically zero. So $b_{12}=0$ and $b_{22}=0$. In other words, only $b_{11}$ in $B$ can be nonzero, in which case we make it nonnegative via a diagonal $T$ to obtain the quadric

$$
w=\left|z_{1}\right|^{2}+\gamma \bar{z}_{1}^{2}, \quad \gamma \geq 0 .
$$

Next let us focus on

$$
A=\left[\begin{array}{ll}
0 & 1 \\
0 & 0
\end{array}\right] .
$$

As above, we compute $\beta^{*} A \beta$ :

$$
\begin{aligned}
& 0= \beta^{*} A \beta=\bar{\beta}_{1} \beta_{2}=\overline{\left(2 \bar{b}_{12} z_{1}+2 \bar{b}_{22} z_{2}\right)}\left(-\bar{z}_{2}-2 \bar{b}_{11} z_{1}-2 \bar{b}_{12} z_{2}\right) \\
&=-2 b_{12} \bar{z}_{1} \bar{z}_{2}-2 b_{22} \bar{b}_{11} z_{1} \bar{z}_{2}-4 \bar{b}_{11} b_{12} z_{1} \bar{z}_{1}-4 b_{12} \bar{b}_{12} \bar{z}_{1} z_{2} \\
&-2 b_{22} \bar{z}_{2}^{2}-4 b_{22} \bar{b}_{12} z_{2} \bar{z}_{2} .
\end{aligned}
$$

Again, as this polynomial must be identically zero, all coefficients must be zero. Hence $b_{12}=0$ and $b_{22}=0$. Again only $b_{11}$ is left possibly nonzero.

Suppose that $b_{11} \neq 0$. Then let $s$ be such that $b_{11} \bar{s}^{2}=1$, and let $\bar{t}=1 / \bar{s}$. The matrix $T=\left[\begin{array}{ll}s & 0 \\ 0 & t\end{array}\right]$ is such that $T^{*} A T=A$ and $T^{*} B \bar{T}=\left[\begin{array}{ll}1 & 0 \\ 0 & 0\end{array}\right]$. If $b_{11}=0$, we have 
$B=0$. Therefore we have obtained two distinct possibilities for $B$, and thus the two submanifolds

$$
w=\bar{z}_{1} z_{2} \quad \text { or } \quad w=\bar{z}_{1} z_{2}+\bar{z}_{1}^{2} .
$$

We emphasize that after $A$ is normalized by a transformation of the form (33), only one coordinate change is needed to normalize $b_{11}$ and this coordinate change preserves $A$. Both are required in a reduction proof for higher dimensions.

We have handled the rank-one case. Next we focus on the rank-two case, that is, $a_{21} \neq 0$ and $a_{12} \neq 0$ (recall $a_{22}=0$ ). We normalize (rescale) $A$ to have $a_{12}=1$ and take

$$
A=\left[\begin{array}{ll}
a_{11} & 1 \\
a_{21} & 0
\end{array}\right]
$$

Again, let us compute $\beta^{*} A \beta$. In the computation for the rank-two case, recall that we have not done any normalization other than rescaling, so we can safely still assume that $b_{22}=0$,

$$
\begin{aligned}
0= & \beta^{*} A \beta=a_{11} \bar{\beta}_{1} \beta_{1}+\bar{\beta}_{1} \beta_{2}+a_{21} \beta_{1} \bar{\beta}_{2} \\
= & a_{11} \overline{\left(\bar{a}_{21} \bar{z}_{1}+2 \bar{b}_{12} z_{1}\right)}\left(\bar{a}_{21} \bar{z}_{1}+2 \bar{b}_{12} z_{1}\right) \\
& \quad+\overline{\left(\bar{a}_{21} \bar{z}_{1}+2 \bar{b}_{12} z_{1}\right)}\left(-\bar{a}_{11} \bar{z}_{1}-\bar{z}_{2}-2 \bar{b}_{11} z_{1}-2 \bar{b}_{12} z_{2}\right) \\
& \quad+a_{21} \overline{\left(-\bar{a}_{11} \bar{z}_{1}-\bar{z}_{2}-2 \bar{b}_{11} z_{1}-2 \bar{b}_{12} z_{2}\right)}\left(\bar{a}_{21} \bar{z}_{1}+2 \bar{b}_{12} z_{1}\right) \\
= & \left(-4\left|b_{12}\right|^{2}-\left|a_{21}\right|^{2}\right) \bar{z}_{1} z_{2}+(\text { other terms }) .
\end{aligned}
$$

All coefficients must be zero. So $a_{21}=0$, and $A$ would not be rank two.

Let us now focus on $n>2$. First suppose that $A=0$. Then as before, $M$ is automatically Levi-flat and by diagonalizing $B$ we obtain the $n$ distinct submanifolds

$$
\begin{aligned}
w & =\bar{z}_{1}^{2}, \\
w & =\bar{z}_{1}^{2}+\bar{z}_{2}^{2}, \\
& \vdots \\
w & =\bar{z}_{1}^{2}+\bar{z}_{2}^{2}+\cdots+\bar{z}_{n}^{2} .
\end{aligned}
$$

Thus suppose from now on that $A \neq 0$. As before, we have an irreducible $(n-1)$-dimensional variety $V \subset M$ through the origin, such that $w=0$ and $A(z, \bar{z})+B(\bar{z}, \bar{z})=0$ on $V$.

We wish to show that $A(z, \bar{z})+B(\bar{z}, \bar{z})=0$ on an $(n-1)$-dimensional linear subspace. For any $\xi \in V$, we obtain $A(z, \bar{\xi})+B(\bar{\xi}, \bar{\xi})=0$ for all $z \in V$. If $V$ is contained in the kernel of the matrix $A^{*}$, then $V$ is a linear subspace of dimension 
$n-1$. So suppose that $\bar{\xi}$ is not in the kernel of the matrix $A^{t}$. Then for a fixed $\bar{\xi}$, we obtain a linear equation $A(z, \bar{\xi})+B(\bar{\xi}, \bar{\xi})=0$ for $z \in V$.

Therefore, as $A(z, \bar{z})+B(\bar{z}, \bar{z})$ needs to be zero on an $(n-1)$-dimensional subspace, we can just make this $\left\{z_{1}=0\right\}$ and so each monomial is divisible by either $z_{1}$ or $\bar{z}_{1}$. Therefore, $A$ and $B$ is of the form

$$
\left[\begin{array}{cccc}
* & * & \cdots & * \\
* & 0 & \cdots & 0 \\
\vdots & \vdots & \ddots & \vdots \\
* & 0 & \cdots & 0
\end{array}\right],
$$

that is, only first column and first row are nonzero. We normalize $A$ via

$$
(A, B) \mapsto\left(\lambda T^{*} A T, \lambda T^{*} B \bar{T}\right),
$$

as before. We use column operations on all but the first column to make all but the first two columns have nonzero elements. Similarly we can do row operations on all but the first two rows to make all but first three rows nonzero. That is, $A$ has the form

$$
\left[\begin{array}{ccccc}
* & * & 0 & \cdots & 0 \\
* & 0 & 0 & \cdots & 0 \\
* & 0 & 0 & \cdots & 0 \\
0 & 0 & 0 & \cdots & 0 \\
\vdots & \vdots & \vdots & \ddots & \vdots \\
0 & 0 & 0 & \cdots & 0
\end{array}\right] .
$$

By Lemma 2.2, setting $z_{3}=\cdots=z_{n}=0$, we obtain a Levi-flat submanifold where the matrix corresponding to $A$ is the principal $2 \times 2$ submatrix of $A$. This submatrix cannot be of rank two and hence either $a_{12}=0$ or $a_{21}=0$. If $a_{21}=0$ and $a_{12} \neq 0$, then setting $z_{2}=z_{3}$ and $z_{4}=\cdots=z_{n}=0$, we again must have a rank-one matrix and therefore $a_{31}=0$.

Therefore, if $a_{12} \neq 0$ then all but $a_{11}$ and $a_{12}$ are zero. If $a_{12}=0$, then via a further linear map not involving $z_{1}$ we can ensure that $a_{31}=0$. In particular, $A$ is of rank one and can only be nonzero in the principal $2 \times 2$ submatrix. At this point $B$ is still of the form (44).

Via a linear change of coordinates in the first two variables, the principal $2 \times 2$ submatrix of $A$ can be normalized into one of the two possible forms

$$
\left[\begin{array}{ll}
1 & 0 \\
0 & 0
\end{array}\right] \text { and }\left[\begin{array}{ll}
0 & 1 \\
0 & 0
\end{array}\right] \text {. }
$$

Recall that $A=0$ was already handled. 
Via the two-dimensional computation we obtain that $b_{22}=b_{12}=b_{21}=0$. We use a linear map in $z_{1}$ and $z_{2}$ to normalize the principal $2 \times 2$ matrix of $B$, so that the submanifold restricted to $\left(z_{1}, z_{2}, w\right)$ is in (B. $\left.\gamma\right),($ C. 0$)$ or (C.1).

Finally we need to show that all entries of $B$ other than $b_{11}$ are zero. As we have done a linear change of coordinates in $z_{1}$ and $z_{2}, B$ may not be in the form (44), but we know $b_{j k}=0$ as long as $j>2$ and $k>2$.

Now fix $k=3, \ldots, n$. Restrict to the submanifold given by $z_{1}=\lambda z_{2}$ for $\lambda=1$ or $\lambda=-1$, and $z_{j}=0$ for all $j=3, \ldots, n$ except for $j=k$. In the variables $\left(z_{2}, z_{k}, w\right)$, we obtain a Levi-flat submanifold where the matrix corresponding to $A$ is $\left[\begin{array}{ll}\lambda & 0 \\ 0 & 0\end{array}\right]$. The matrix corresponding to $B$ is

$$
\left[\begin{array}{cc}
b_{11} & b_{1 k}+\lambda b_{2 k} \\
b_{1 k}+\lambda b_{2 k} & 0
\end{array}\right] .
$$

Via the two-dimensional calculation we have $b_{1 k}+\lambda b_{2 k}=0$. As this is true for $\lambda=1$ and $\lambda=-1$, we get that $b_{1 k}=b_{2 k}=0$.

We have proved the following classification result. It is not difficult to see that the submanifolds in the list are biholomorphically inequivalent by Proposition 2.1. The ranks of $A$ and $B$ are invariants. It is obvious that the $A$ matrix of B. $\gamma$ and C.x submanifolds are inequivalent. Therefore, it is only necessary to directly check that B. $\gamma$ are inequivalent for different $\gamma \geq 0$, which is easy.

Lemma 3.2. If $M$ defined in $(z, w) \in \mathbb{C}^{n} \times \mathbb{C}, n \geq 1$, by

$$
w=A(z, \bar{z})+B(\bar{z}, \bar{z})
$$

is Levi-flat, then $M$ is biholomorphic to one and exactly one of the expressions (A.1)-(C.1) of Theorem 1.1.

The normalizing transformation used above is linear.

Lemma 3.3. If $M$, defined by

$$
w=A(z, \bar{z})+B(\bar{z}, \bar{z})+O(3),
$$

is Levi-flat at all points where $M$ is $C R$, then the quadric

$$
w=A(z, \bar{z})+B(\bar{z}, \bar{z})
$$

is also Levi-flat.

Proof. Write $M$ as

$$
w=A(z, \bar{z})+B(\bar{z}, \bar{z})+r(z, \bar{z}),
$$

where $r$ is $O(3)$.

Let $A$ be the matrix giving the quadratic form $A(z, \bar{z})$ as before. The Levi map is given by taking the $n \times n$ matrix 


$$
L=L(p)=A+\left[\frac{\partial^{2} r}{\partial z_{j} \partial \bar{z}_{k}}\right]_{j, k}
$$

and applying it to vectors in $\pi\left(T^{(1,0)} M\right)$, where $\pi$ is the projection onto the $\{w=0\}$ plane. That is, we parametrize $M$ by the $\{w=0\}$ plane, and work there as before.

Let

$$
\begin{aligned}
a_{j} & =-\bar{A}_{z_{j}}-\bar{B}_{z_{j}}-\bar{r}_{z_{j}}, \\
b & =\bar{A}_{z_{1}}+\bar{B}_{z_{1}}+\bar{r}_{z_{1}}, \\
c & =a_{j}\left(A_{z_{1}}+B_{z_{1}}+r_{z_{1}}\right)+b\left(A_{z_{j}}+B_{z_{j}}+r_{z_{j}}\right) .
\end{aligned}
$$

Then for $j=2, \ldots, n$, we write the $T^{(1,0)}$ vector fields as

$$
X_{j}=a_{j} \frac{\partial}{\partial z_{1}}+b \frac{\partial}{\partial z_{j}}+c \frac{\partial}{\partial w} .
$$

Hence

$$
a_{j} \frac{\partial}{\partial z_{1}}+b \frac{\partial}{\partial z_{j}}
$$

are the vector fields in $\pi\left(T^{(1,0)} M\right)$.

Notice that $a_{j}, b$, and $c$ vanish at the origin. Furthermore, if we take the linear terms of $a_{j}, b$, and the quadratic terms in $c$, that is,

$$
\begin{aligned}
\tilde{a}_{j} & =-\bar{A}_{z_{j}}-\bar{B}_{z_{j}}, \\
\tilde{b} & =\bar{A}_{z_{1}}+\bar{B}_{z_{1}}, \\
\tilde{c} & =\tilde{a}_{j}\left(A_{z_{1}}+B_{z_{1}}\right)+\tilde{b}\left(A_{z_{j}}+B_{z_{j}}\right),
\end{aligned}
$$

then away from the CR singular set of the quadric,

$$
\tilde{X}_{j}=\tilde{a}_{j} \frac{\partial}{\partial z_{1}}+\tilde{b} \frac{\partial}{\partial z_{j}}+\tilde{c} \frac{\partial}{\partial w}
$$

span the $T^{(1,0)}$ vector fields on the quadric $w=A(z, \bar{z})+B(\bar{z}, \bar{z})$.

Since $M$ is Levi-flat, then we have that

$$
\pi_{*}\left(X_{j}\right)^{*} L \pi_{*}\left(X_{j}\right)=0 .
$$

The terms linear in $z$ and $\bar{z}$ respectively in the expression $\pi_{*}\left(X_{j}\right)^{*} L \pi_{*}\left(X_{j}\right)$ are

$$
\pi_{*}\left(\tilde{X}_{j}\right)^{*} A \pi_{*}\left(\tilde{X}_{j}\right) .
$$

As this is identically zero, the quadric $w=A(z, \bar{z})+B(\bar{z}, \bar{z})$ is Levi-flat. 


\section{Quadratic Levi-flat submanifolds and their Segre varieties}

A very useful invariant in CR geometry is the Segre variety. Suppose that a realanalytic variety $X \subset \mathbb{C}^{N}$ is defined by

$$
\rho(z, \bar{z})=0,
$$

where $\rho$ is a real-analytic real vector-valued with $p \in X$. Suppose that $\rho$ converges on some polydisc $\Delta$ centered at $p$. We complexify and treat $z$ and $\bar{z}$ as independent variables, and the power series of $\rho$ at $(p, \bar{p})$ converges on $\Delta \times \Delta$. The Segre variety at $p$ is then defined as the variety

$$
Q_{p}=\{z \in \Delta: \rho(z, \bar{p})=0\} .
$$

Of course the variety depends on the defining equation itself and the polydisc $\Delta$. For $\rho$, it is useful to take the defining equation or equations that generate the ideal of the complexified $X$ in $\mathbb{C}^{N} \times \mathbb{C}^{N}$ at $p$. If $\rho$ is polynomial we take $\Delta=\mathbb{C}^{N}$.

It is well known that any irreducible complex variety that lies in $X$ and goes through the point $p$ also lies in $Q_{p}$. In the case of Levi-flat submanifolds, we generally get equality as germs. For example, for the CR Levi-flat submanifold $M$ given by

$$
\operatorname{Im} z_{1}=0, \quad \operatorname{Im} z_{2}=0,
$$

the Segre variety $Q_{0}$ through the origin is precisely $\left\{z_{1}=z_{2}=0\right\}$, which happens to be the unique complex variety in $M$ through the origin.

Let us take the Levi-flat quadric

$$
w=A(z, \bar{z})+B(\bar{z}, \bar{z}) .
$$

As we want to take the generating equations in the complexified space we also need the conjugate

$$
\bar{w}=\bar{A}(\bar{z}, z)+\bar{B}(z, z) .
$$

The Segre variety is then given by

$$
w=0, \quad \bar{B}(z, z)=0 .
$$

Through any CR singular point of a real-analytic Levi-flat $M$, there is a complex variety of dimension $n-1$ that is the limit of the leaves of the Levi foliation of $M_{\mathrm{CR}}$, via Lemma 3.1. Let us take all possible such limits, and call their union $Q_{p}^{\prime}$. Notice that there could be other complex varieties in $M$ through $p$ of dimension $n-1$. Note that $Q_{p}^{\prime} \subset Q_{p}$.

Let us write down and classify the Segre varieties for all the quadric Levi-flat submanifolds in $\mathbb{C}^{n+1}$ : 


\begin{tabular}{c|c|c|c|c|c} 
Type & Segre variety $Q_{0}$ & $Q_{0}$ singular? & $\operatorname{dim}_{\mathbb{C}} Q_{0}$ & $Q_{0} \subset M ?$ & $Q_{0}^{\prime}$ \\
\hline A.1 & $w=0, z_{1}^{2}=0$ & no & $n-1$ & yes & $Q_{0}$ \\
A. $k$ & $\begin{array}{c}w=0 \\
z_{1}^{2}+\cdots+z_{k}^{2}=0\end{array}$ & yes & $n-1$ & yes & $Q_{0}$ \\
B.0 & $w=0$ & no & $n$ & no & $w=0$ \\
B. $\gamma, \gamma>0$ & $w=0, z_{1}^{2}=0$ & no & $n-1$ & yes & $Q_{0}=0$ \\
C. 0 & $w=0$ & no & $n$ & no & $\begin{array}{c}w=0 \\
z_{1}=0 \\
\text { C. } 1\end{array}$ \\
$w=0, z_{1}^{2}=0$ & no & $n-1$ & yes & $Q_{0}$
\end{tabular}

The submanifold C. 0 also contains the complex variety $\left\{w=0, z_{2}=0\right\}$, but this variety is transversal to the leaves of the foliation, and so cannot be in $Q_{0}^{\prime}$

Notice that in the cases A. $k$ for all $k$, B. $\gamma$ for $\gamma>0$, and C.1, the variety $Q_{0}$ actually gives the complex variety $Q_{0}^{\prime}$ contained in $M$ through the origin. In these cases, the variety is nonsingular only in the set theoretic sense. Scheme-theoretically the variety is always at least a double line or double hyperplane in general.

\section{The CR singularity of Levi-flat quadrics}

Proposition 5.1. Let $M \subset \mathbb{C}^{n+1}$ be given by

$$
w=\rho(z, \bar{z}),
$$

where $\rho$ is $O(2)$, and $M$ is not a complex submanifold. Then the set $S$ of $C R$ singularities of $M$ is given by

$$
S=\{(z, w): \bar{\partial} \rho=0, w=\rho(z, \bar{z})\} .
$$

Proof. This is well-known; we give a proof for convenience. In codimension two, a real submanifold is either CR singular, complex, or generic. A submanifold is generic if $\bar{\partial}$ of all the defining equations are pointwise linearly independent (see [Baouendi et al. 1999]). As $M$ is not complex, to find the set of CR singularities, we find the set of points where $M$ is not generic. We need both defining equations for $M$,

$$
w=\rho(z, \bar{z}) \quad \text { and } \quad \bar{w}=\rho(z, \bar{z}) .
$$

As the second equation always produces a $d \bar{w}$ while the first does not, the only way that the two can be linearly dependent is for the $\bar{\partial}$ of the first equation to be zero. In other words, $\bar{\partial} \rho=0$.

Let us compute and classify the CR singular sets for the CR singular Levi-flat quadrics: 


\begin{tabular}{c|c|c|c} 
Type & CR singularity $S$ & $\operatorname{dim}_{\mathbb{R}} S$ & CR structure of $S$ \\
\hline A. $k$ & $z_{1}=\cdots=z_{k}=0, w=0$ & $2 n-2 k$ & complex \\
B. 0 & $z_{1}=0, w=0$ & $2 n-2$ & complex \\
B. $\frac{1}{2}$ & $z_{1}+\bar{z}_{1}=0, w=0$ & $2 n-1$ & Levi-flat \\
B. $\gamma, \gamma>0, \gamma \neq \frac{1}{2}$ & $z_{1}=0, w=0$ & $2 n-2$ & complex \\
C. 0 & $z_{2}=0, w=0$ & $2 n-2$ & complex \\
C. 1 & $z_{2}+2 \bar{z}_{1}=0, w=-\frac{1}{4} z_{2}^{2}$ & $2 n-2$ & Levi-flat
\end{tabular}

By Levi-flat we mean that $S$ is a Levi-flat CR submanifold in $\{w=0\}$. There is a conjecture that a real subvariety that is Levi-flat at CR points has a stratification by Levi-flat CR submanifolds. This computation gives further evidence of this conjecture.

\section{Levi foliations and images of generic Levi-flats}

A CR Levi-flat submanifold $M \subset \mathbb{C}^{n}$ of codimension two has a certain canonical foliation defined on it with complex analytic leaves of real codimension two in $M$. The submanifold $M$ is locally equivalent to $\mathbb{R}^{2} \times \mathbb{C}^{n-2}$, defined by

$$
\operatorname{Im} z_{1}=0, \quad \operatorname{Im} z_{2}=0 .
$$

The leaves of the foliation are the submanifolds given by fixing $z_{1}$ and $z_{2}$ at a real constant. By foliation we always mean the standard nonsingular foliation as locally comes up in the implicit function theorem. This foliation on $M$ is called the Levi foliation. It is obvious that the Levi foliation on $M$ extends to a neighborhood of $M$ as a nonsingular holomorphic foliation. The same is not true in general for CR singular submanifolds. We say that a smooth holomorphic foliation $\mathcal{L}$ defined in a neighborhood of $M$ is an extension of the Levi foliation of $M_{\mathrm{CR}}$, if $\mathcal{L}$ and the Levi foliation have the same germs of leaves at each CR point of $M$. We also say that a smooth real-analytic foliation $\widetilde{\mathcal{L}}$ on $M$ is an extension of the Levi foliation on $M_{\mathrm{CR}}$ if $\widetilde{\mathcal{L}}$ and the Levi foliation have the same germs of leaves at each CR point of $M$. In our situation (real-analytic), $M_{\mathrm{CR}}$ is a dense and open subset of $M$. This implies that the leaves of $\mathcal{L}$ and $\widetilde{\mathcal{L}}$ through a $\mathrm{CR}$ singular point are complex analytic submanifolds contained in $M$. The latter could lead to an obvious obstruction to extension. First let us see what happens if the foliation of $M_{\mathrm{CR}}$ is the restriction of a nonsingular holomorphic foliation of a whole neighborhood of $M$.

The Bishop-like quadrics, that is, A. 1 and B. $\gamma$ in $\mathbb{C}^{n+1}$, have a Levi foliation that extends as a holomorphic foliation to all of $\mathbb{C}^{n+1}$. That is because these submanifolds are of the form

$$
N \times \mathbb{C}^{n-1}
$$


For submanifolds of the form (71) we can find normal forms using the welldeveloped theory of Bishop surfaces in $\mathbb{C}^{2}$.

Proposition 6.1. Suppose $M \subset \mathbb{C}^{n+1}$ is a real-analytic Levi-flat $C R$ singular submanifold where the Levi foliation on $M_{\mathrm{CR}}$ extends near $p \in M$ to a nonsingular holomorphic foliation of a neighborhood of $p$ in $\mathbb{C}^{n+1}$. Then at $p, M$ is locally biholomorphically equivalent to a submanifold of the form

$$
N \times \mathbb{C}^{n-1}
$$

where $N \subset \mathbb{C}^{2}$ is a $C R$ singular submanifold of real dimension two. Therefore if $M$ has a nondegenerate complex tangent, then it is Bishop-like, i.e., of type A.1 or B. $\gamma$.

Furthermore, two submanifolds of the form (72) are locally biholomorphically (resp. formally) equivalent if and only if the corresponding $N \mathrm{~s}$ are locally biholomorphically (resp. formally) equivalent in $\mathbb{C}^{2}$.

Proof. We flatten the holomorphic foliation near $p$ so that in some polydisc $\Delta$, the leaves of the foliation are given by $\{q\} \times \mathbb{C}^{n-1} \cap \Delta$ for $q \in \mathbb{C}^{2}$. Let us suppose that $M$ is closed in $\Delta$. At any CR point of $M$, the leaf of the Levi foliation agrees with that of the holomorphic foliation and therefore the leaf that lies in $M$ agrees with a leaf of the form $\{q\} \times \mathbb{C}^{n-1}$ as a germ and so $\{q\} \times \mathbb{C}^{n-1} \cap \Delta \subset M$. As $M_{\mathrm{CR}}$ is dense in $M$, then $M$ is a union of sets of the form $\{q\} \times \mathbb{C}^{n-1} \cap \Delta$ and the first part follows.

It is classical that every Bishop surface (two-dimensional real submanifold of $\mathbb{C}^{2}$ with a nondegenerate complex tangent) is equivalent to a submanifold whose quadratic part is of the form A. 1 or B. $\gamma$.

Finally, the proof that two submanifolds of the form (72) are equivalent if and only if the $N$ s are equivalent is straightforward.

Not every Bishop-like submanifold is a cross product as above. In fact the Bishop invariant may well change from point to point. See Section 15. In such cases the foliation does not extend to a nonsingular holomorphic foliation of a neighborhood.

Let us now focus on extending the Levi foliation to $M$, and not to a neighborhood of $M$. Let us prove a useful proposition about recognizing certain CR singular Levi-flats from the form of the defining equation. That is, if the $r$ in the equation does not depend on $\bar{z}_{2}$ through $\bar{z}_{n}$.

Proposition 6.2. Suppose that near the origin $M \subset \mathbb{C}^{n+1}$ is given by

$$
w=r\left(z_{1}, \bar{z}_{1}, z_{2}, z_{3}, \ldots, z_{n}\right),
$$

where $r$ is $O(2)$ and $\partial r / \partial \bar{z}_{1} \not \equiv 0$. Then $M$ is a $C R$ singular Levi-flat submanifold and the Levi foliation of $M_{\mathrm{CR}}$ extends through the origin to a real-analytic foliation on $M$. Furthermore, there exists a real-analytic CR mapping $F: U \subset \mathbb{R}^{2} \times \mathbb{C}^{n-1} \rightarrow$ $\mathbb{C}^{n+1}, F(0)=0$, which is a diffeomorphism onto its image $F(U) \subset M$. 
Near zero, $M$ is the image of a CR mapping that is a diffeomorphism onto its image of the standard CR Levi-flat. The proposition also holds in two dimensions $(n=1)$, although in this case it is somewhat trivial.

Proof. As in [Lebl et al. 2014], let us define the mapping $F$ by

$$
(x, y, \xi) \mapsto(x+i y, \xi, r(x+i y, x-i y, \xi)),
$$

where $\xi=\left(\xi_{2}, \ldots, \xi_{n}\right) \in \mathbb{C}^{n-1}$. Near points where $M$ is $\mathrm{CR}$, this mapping is a $\mathrm{CR}$ diffeomorphism and hence $M$ must be Levi-flat. Furthermore, since $F$ is a diffeomorphism, it takes the Levi foliation on $\mathbb{R}^{2} \times \mathbb{C}^{n-1}$ to a foliation on $M$ near zero.

Lemma 6.3. Let $M \subset \mathbb{C}^{n+1}$ be a $C R$ singular real-analytic Levi-flat submanifold of codimension two through the origin.

Then $M$ is a CR singular Levi-flat submanifold whose Levi foliation of $M_{\mathrm{CR}}$ extends through the origin to a nonsingular real-analytic foliation on $M$ if and only if there exists a real-analytic CR mapping $F: U \subset \mathbb{R}^{2} \times \mathbb{C}^{n-1} \rightarrow \mathbb{C}^{n+1}, F(0)=0$, which is a diffeomorphism onto its image $F(U) \subset M$.

Proof. One direction is easy and was used above. For the other direction, suppose that we have a foliation extending the Levi foliation through the origin. Let us consider $M_{\mathrm{CR}}$ an abstract CR manifold. That is a manifold $M_{\mathrm{CR}}$ together with the bundle $T^{(0,1)} M_{\mathrm{CR}} \subset \mathbb{C} \otimes T M_{\mathrm{CR}}$. The extended foliation on $M$ gives a real-analytic subbundle $\mathcal{W} \subset T M$. Since we are extending the Levi foliation, when $p \in M_{\mathrm{CR}}$, then $\mathcal{W}_{p}=T_{p}^{c} M$, where $T_{p}^{c} M=J\left(T_{p}^{c} M\right)$ is the complex tangent space and $J$ is the complex structure on $\mathbb{C}^{n+1}$. Since $M_{\mathrm{CR}}$ is dense in $M$, then $J \mathcal{W}=\mathcal{W}$ on $M$.

Define the real-analytic subbundle $\mathcal{V} \subset \mathbb{C} \otimes T M$ as

$$
\mathcal{V}_{p}=\left\{X+i J(X): X \in \mathcal{W}_{p}\right\}
$$

At CR points $\mathcal{V}_{p}=T_{p}^{(0,1)} M$ (see, for example, [Baouendi et al. 1999, p. 8]). Then we can find vector fields $X^{1}, \ldots, X^{n-1}$ in $\mathcal{W}$ such that

$$
X^{1}, J\left(X^{1}\right), X^{2}, J\left(X^{2}\right), \ldots, X^{n-1}, J\left(X^{n-1}\right)
$$

is a basis of $\mathcal{W}$ near the origin. Then the basis for $\mathcal{V}$ is given by

$$
X^{1}+i J\left(X^{1}\right), X^{2}+i J\left(X^{2}\right), \ldots, X^{n-1}+i J\left(X^{n-1}\right) .
$$

As the subbundle is integrable, we obtain that $(M, \mathcal{V})$ gives an abstract CR manifold, which at CR points agrees with $M_{\mathrm{CR}}$. This manifold is Levi-flat as it is Levi-flat on a dense open set. As it is real-analytic, it is embeddable; and hence there exists a realanalytic CR diffeomorphism from a neighborhood of $\mathbb{R}^{2} \times \mathbb{C}^{n-1}$ to a neighborhood of zero in $M$ (as an abstract CR manifold). This is our mapping $F$. 
The quadrics A. $k, k \geq 2$, defined by

$$
w=\bar{z}_{1}^{2}+\cdots+\bar{z}_{k}^{2},
$$

contain the singular variety defined by $w=0, z_{1}^{2}+\cdots+z_{k}^{2}=0$, and hence the Levi foliation cannot extend to a nonsingular foliation of the submanifold. The quadric A.1 does admit a holomorphic foliation, but other type A.1 submanifolds do not in general. For example, the submanifold

$$
w=\bar{z}_{1}^{2}+\bar{z}_{2}^{3}
$$

is of type A. 1 and the unique complex variety through the origin is $0=z_{1}^{2}+z_{2}^{3}$, which is singular. Therefore the foliation cannot extend to $M$.

\section{Extending the Levi foliation of type C.x submanifolds}

Let us prove Theorem 1.2, that is, let us start with a type C. 0 or C. 1 submanifold and show that the Levi foliation must extend real-analytically to all of $M$. Equivalently, we show that the real-analytic bundle $T^{(1,0)} M_{\mathrm{CR}}$ extends to a real-analytic subbundle of $\mathbb{C} \otimes T M$. Taking real parts we obtain an involutive subbundle of $T M$ extending $T^{c} M_{\mathrm{CR}}=\operatorname{Re}\left(T^{(1,0)} M_{\mathrm{CR}}\right)$.

Proof of Theorem 1.2. Let $M$ be the submanifold given by

$$
w=\bar{z}_{1} z_{2}+\epsilon \bar{z}_{1}^{2}+r(z, \bar{z}),
$$

where $\epsilon=0,1$. Let us treat the $z$ variables as the parameters on $M$. Let $\pi$ be the projection onto the $\{w=0\}$ plane, which is tangent to $M$ at zero as a real $2 n$ dimensional hyperplane. We will look at all the vector fields on this plane $\{w=0\}$. All vectors in $\pi\left(T^{(1,0)} M\right)$ can be written in terms of $\partial / \partial z_{j}$ for $j=1, \ldots, n$.

The Levi map is given by taking the $n \times n$ matrix

$$
L=L(p)=\left[\begin{array}{ccccc}
0 & 1 & 0 & \cdots & 0 \\
0 & 0 & 0 & \cdots & 0 \\
0 & 0 & 0 & \cdots & 0 \\
\vdots & \vdots & \vdots & \ddots & \vdots \\
0 & 0 & 0 & \cdots & 0
\end{array}\right]+\left[\frac{\partial^{2} r}{\partial z_{j} \partial \bar{z}_{k}}\right]_{j, k}(p)
$$

to vectors $v \in \pi\left(T^{(1,0)} M\right)$ ( $\pi$ is the projection) as $v^{*} L v$. The excessive term in $L$ vanishes at zero.

Notice that for $p \in M_{\mathrm{CR}}, \pi\left(T_{p}^{(1,0)} M\right)$ is $(n-1)$-dimensional. As $M$ is Levi-flat, then $v^{*} L v$ vanishes for $v \in \pi\left(T_{p}^{(1,0)} M\right)$. Write the vector $v=\left(v_{1}, \ldots, v_{n}\right)^{t}$. The zero set of the function

$$
(z, v) \in \mathbb{C}^{n} \times \mathbb{C}^{n} \stackrel{\varphi}{\mapsto} v^{*} L(z, \bar{z}) v
$$


is a variety $V$ of real codimension two at the origin of $\mathbb{C}^{n} \times \mathbb{C}^{n}$ because of the form of $L$. That is, at $z=0$, the only vectors $v$ such that $v^{*} L v=0$ are those where $v_{1}=0$ or $v_{2}=0$. So the codimension is at least two. And we know that $v^{*} L v$ vanishes for vectors in $\pi\left(T_{p}^{(1,0)} M\right)$ for $p \in M$ near zero, which is real codimension two at each $z$ corresponding to a CR point. Therefore, $V \cap\left(\pi\left(M_{\mathrm{CR}}\right) \times \mathbb{C}^{n}\right)$ has a connected component that is equal to a connected component of the real-analytic subbundle $\pi\left(T^{(1,0)} M_{\mathrm{CR}}\right)$. We will verify that the latter is connected.

We show below that this subbundle extends past the CR singularity. The key point is to show that the restriction of $\pi\left(T^{(1,0)}\left(M_{\mathrm{CR}}\right)\right)$ extends to a smooth real-analytic submanifold of $T^{(1,0)} \mathbb{C}^{n}$. Write

$$
\varphi(z, v)=v_{1} \bar{v}_{2}+\sum a_{j k}(z) v_{j} \bar{v}_{k},
$$

where $a_{j k}(0)=0$.

By Proposition 5.1, $\pi\left(M \backslash M_{\mathrm{CR}}\right)$ is contained in

$$
z_{2}+2 \epsilon \bar{z}_{1}+r_{\bar{z}_{1}}=0 \text {. }
$$

Thus $M_{\mathrm{CR}}$ is connected. Assume that $v \cdot(\partial / \partial z) \in T_{p}^{(1,0)} M$ at a CR point $p$. Then

$$
\left(z_{2}+2 \epsilon \bar{z}_{1}+r_{\bar{z}_{1}}\right) \bar{v}_{1}+\sum_{j>1} r_{\bar{z}_{j}} \bar{v}_{j}=0 .
$$

When $p$ is in the open set $U_{\delta} \subset \pi\left(M_{\mathrm{CR}}\right)$ defined by $\left|z_{2}+2 \epsilon \bar{z}_{1}\right|>\frac{1}{2}|z|$ and $0<|z|<\delta, v$ is contained in

$$
V_{C}:\left|v_{1}\right| \leq|v| / C .
$$

When $\delta$ is sufficiently small, $\varphi(z, v)=0$ admits a unique solution

$$
v_{1}=f\left(z, v_{3}, \ldots, v_{n}\right), \quad v_{2}=1
$$

by imposing $v \in V_{C}$. Note that $f$ is given by convergent power series. For $|z|<\delta$, define

$$
w_{j}=\left(w_{j 1}(z), \ldots, w_{j n}(z)\right) \in V_{C}, \quad j=2, \ldots, n
$$

such that $\varphi\left(z, w_{j}(z)\right)=0$ and

$$
w_{j 2}=1, \quad w_{j k}=\delta_{j k}, \quad j \geq 2, k>2 .
$$

To see why we can do so, fix $p \in U_{\delta}$. First we can find a vector $w_{2}$ in $E_{p}=$ $\pi\left(T_{p}^{(1,0)} M_{\mathrm{CR}}\right)$ such that $v_{2}=1$. Otherwise, $E_{p} \subset V_{C}$ cannot have dimension $n-1$. Let $E_{p}^{\prime}$ be the vector subspace of $E_{p}$ with $v_{2}=0$. Then $E_{p}^{\prime}$ has rank $n-2$ and remains in the cone $V_{C}$. Then $E_{p}^{\prime}$ has an element $w_{2}$ with $v_{2}$ component being one. Repeating this, we find $w_{2}, \ldots, w_{n}$ in $E_{p}$ such that the $v_{j}$ component of $w_{i}$ is zero 
for $2<j<i$. Using linear combinations, we find a unique basis $\left\{w_{2}, \ldots, w_{n}\right\}$ of $E_{p}$ that satisfies condition (89).

Assume that $C$ is sufficiently large. By the above uniqueness assertion on $\varphi(z, v)=0$, we conclude that when $p \in U_{\delta},\left\{w_{2}(p), \ldots, w_{n}(p)\right\}$ is a basis of $\pi\left(T_{p}^{(1,0)} M_{\mathrm{CR}}\right)$. Also it is real analytic at $p=0$. Define

$$
\omega_{j}(z)=w_{j}(z) \cdot \frac{\partial}{\partial z}, \quad|z|<\delta .
$$

We lift the functions $\omega_{j}$ via $\pi$ to a subbundle of $\mathbb{C} \otimes T M$; let us call them $\widetilde{\omega}_{j}$. Then consider the vector fields $w_{j}^{*}=2 \operatorname{Re} \widetilde{\omega}_{j}=\widetilde{\omega}_{j}+\widetilde{\widetilde{\omega}}_{j}$ and $w_{n+j}^{*}=\operatorname{Im} \widetilde{\omega}_{j}$ for $j=2, \ldots, n$. Above CR points over $U_{\delta}, \widetilde{w}_{j}$ is in $T M_{\mathrm{CR}} \otimes \mathbb{C}$ and so tangent to $M$. We thus obtain a $(2 n-2)$-dimensional real-analytic subbundle of $T M$ that agrees with the real-analytic real subbundle of $T M_{\mathrm{CR}}$ induced by the Levi foliation above $U_{\delta}$. Since $M_{\mathrm{CR}}$ and the subbundles are real analytic and $M_{\mathrm{CR}}$ is connected, they agree over $M_{\mathrm{CR}}$.

The real-analytic distribution spanned by $\left\{\omega_{i}^{*}\right\}$ has constant rank $2 n-2$ everywhere and is involutive on an open subset of $M_{\mathrm{CR}}$ and hence everywhere.

\section{CR singular set of type C.x submanifolds}

Let $M \subset \mathbb{C}^{n+1}$ be a codimension-two Levi-flat $\mathrm{CR}$ singular submanifold that is an image of $\mathbb{R}^{2} \times \mathbb{C}^{n-1}$ via a real-analytic CR map, and let $S \subset M$ be the CR singular set of $M$. In [Lebl et al. 2014] it was proved that near a generic point of $S$, exactly one of the following is true:

(i) $S$ is a Levi-flat submanifold of dimension $2 n-2$ and CR dimension $n-2$.

(ii) $S$ is a complex submanifold of complex dimension $n-1$ (real dimension $2 n-2)$.

(iii) $S$ is a Levi-flat submanifold of dimension $2 n-1$ and CR dimension $n-1$.

We only have the above classification for a generic point of $S$, and $S$ need not be a CR submanifold everywhere. See [Lebl et al. 2014] for examples.

If $M$ is a Levi-flat CR singular submanifold and the Levi foliation of $M_{\mathrm{CR}}$ extends to $M$, then by Lemma 6.3 at a generic point $S$ has to be of one of the above types. A corollary of Theorem 1.2 is:

Corollary 8.1. Suppose that $M \subset \mathbb{C}^{n+1}, n \geq 2$, is a real-analytic Levi-flat $C R$ singular type $C .1$ or type $C .0$ submanifold. Let $S \subset M$ denote the $C R$ singular set. Then near the origin $S$ is a submanifold of dimension $2 n-2$, and at a generic point, $S$ is either CR Levi-flat of dimension $2 n-2(C R$ dimension $n-2)$ or a complex submanifold of complex dimension $n-1$.

Furthermore, if $M$ is of type C.1, then at the origin $S$ is a CR Levi-flat submanifold of dimension $2 n-2$ (CR dimension $n-2)$. 
Proof. Let $M$ be given by

$$
w=\bar{z}_{1} z_{2}+\epsilon \bar{z}_{1}^{2}+r(z, \bar{z}),
$$

where $r$ is $O(3)$ and $\epsilon=0$ or $\epsilon=1$.

By Proposition 5.1 the CR singular set is exactly where

$$
z_{2}+\epsilon 2 \bar{z}_{1}+r_{\bar{z}_{1}}(z, \bar{z})=0 \quad \text { and } \quad r_{\bar{z}_{j}}(z, \bar{z})=0 \quad \text { for all } j=2, \ldots, n \text {. }
$$

By considering the real and imaginary parts of the first equation and applying the implicit function theorem, the set $\widetilde{S}=\left\{z: z_{2}+\epsilon 2 \bar{z}_{1}+r_{\bar{z}_{1}}(z, \bar{z})=0\right\}$ is a real submanifold of real dimension $2 n-2$ (real codimension two in $M$ ). Now $S \subset \widetilde{S}$, but as we saw above that $S$ is of dimension at least $2 n-2$. Therefore $S=\widetilde{S}$ near the origin. The conclusion of the first part then follows from the classification above.

The stronger conclusion for type C.1 submanifolds follows by noticing that when $\epsilon=1$, the submanifold

$$
z_{2}+2 \bar{z}_{1}+r_{\bar{z}_{1}}(z, \bar{z})=0
$$

is $\mathrm{CR}$ and not complex at the origin.

\section{Mixed-holomorphic submanifolds}

Let us study sets in $\mathbb{C}^{m}$ defined by

$$
f\left(\bar{z}_{1}, z_{2}, \ldots, z_{m}\right)=0
$$

for a single holomorphic function $f$ of $m$ variables.

Such sets have much in common with complex varieties, since they are in fact complex varieties when $\bar{z}_{1}$ is treated as a complex variable. The distinction is that the automorphism group is different since we are interested in automorphisms that are holomorphic, not mixed-holomorphic.

Proposition 9.1. If $M \subset \mathbb{C}^{m}$ is a submanifold with a defining equation of the form (94), where $f$ is a holomorphic function that is not identically zero, then $M$ is a real codimension-two set and $M$ is either a complex submanifold or a Levi-flat submanifold, possibly $C R$ singular. Furthermore, if $M$ is $C R$ singular at $p \in M$, and has a nondegenerate complex tangent at $p$, then $M$ has type A.k, C.0, or C.1 at $p$.

Proof. Since the zero set of $f$ is a complex variety in the $\left(\bar{z}_{1}, z_{2}, \ldots, z_{m}\right)$ space, we get automatically that it is real codimension two. We also have that as it is a submanifold, then it can be written as a graph of one variable over the rest.

Let $m=n+1$ for convenience and suppose that $M \subset \mathbb{C}^{n+1}$ is a submanifold through the origin. By factorization for germs of holomorphic functions and by the 
smoothness assumption on $M$ we may assume that $d f(0) \neq 0$. Call the variables $\left(z_{1}, \ldots, z_{n}, w\right)$ and write $M$ as a graph. One possibility is that we write $M$ as

$$
\bar{w}=\rho\left(z_{1}, \ldots, z_{n}\right),
$$

where $\rho(0)=0$ and $\rho$ has no linear terms. $M$ is complex if $\rho \equiv 0$. Otherwise $M$ is $\mathrm{CR}$ singular and we rewrite it as

$$
w=\bar{\rho}\left(\bar{z}_{1}, \ldots, \bar{z}_{n}\right) .
$$

We notice that the matrix representing the Levi-map must be identically zero, so we must get Levi-flat. If there are any quadratic terms, we obtain a type A.k submanifold.

Alternatively, $M$ can be written as

$$
w=\rho\left(\bar{z}_{1}, z_{2}, \ldots, z_{n}\right),
$$

with $\rho(0)=0$. If $\rho$ does not depend on $\bar{z}_{1}$ then $M$ is complex. Assume that $\rho$ depends on $\bar{z}_{1}$. If $\rho$ has linear terms in $\bar{z}_{1}$, then $M$ is CR. Otherwise it is a CR singular submanifold, and near a nonCR singular point it is a generic codimensiontwo submanifold. The CR singular set of $M$ is defined by $\partial \rho / \partial \bar{z}_{1}=0$.

Suppose that $M$ is CR singular. That $M$ is Levi-flat follows from Proposition 6.2. We can therefore normalize the quadratic term, after linear terms in $z_{2}, \ldots, z_{n}$ are absorbed into $w$.

If not all quadratic terms are zero, we must have an A.k, C.0, or C.1 type submanifold.

Let us now study normal forms for such sets in $\mathbb{C}^{2}$ and $\mathbb{C}^{m}, m \geq 3$. First in two variables we can completely answer the question. This result is surely well known and classical.

Proposition 9.2. If $M \subset \mathbb{C}^{2}$ is a submanifold with a defining equation of the form (94), then it is locally biholomorphically equivalent to a submanifold in coordinates $(z, w) \in \mathbb{C}^{2}$ of the form

$$
w=\bar{z}^{d}
$$

for $d=0,1,2,3, \ldots$, where $d$ is a local biholomorphic invariant of $M$. If $d=0$, $M$ is complex; if $d=1$, it is a CR totally-real submanifold; and if $d \geq 2$, then $M$ is CR singular.

Proof. Write the submanifold as a graph of one variable over the other. Without loss of generality and after possibly taking a conjugate of the equation, we have

$$
w=f(\bar{z})
$$


for some holomorphic function $f$. Assume $f(0)=0$. If $f$ is identically zero, then $d=0$ and we are finished. If $f$ is not identically zero, we apply a holomorphic change of coordinates in $z$, and the rest follows easily.

In three or more variables, if $M \subset \mathbb{C}^{n+1}, n \geq 2$, is a submanifold through the origin, then when the quadratic part is nonzero we have seen above that it can be a type A. $k$, C. 0 , or C.1 submanifold. If the submanifold is the nondegenerate type C.1 submanifold, then we will show in the next section that $M$ is biholomorphically equivalent to the quadric $M_{C .1}$.

Before we move to type C.1, let us quickly consider the mixed-holomorphic submanifolds of type A.n. The submanifolds of type A. $n$ in $\mathbb{C}^{n+1}$ can in some sense be considered nondegenerate when talking about mixed-holomorphic submanifolds. Proposition 9.3. If $M \subset \mathbb{C}^{n+1}$ is a submanifold of type A.n at the origin of the form

$$
w=\bar{z}_{1}^{2}+\cdots+\bar{z}_{n}^{2}+r(\bar{z}),
$$

where $r \in O(3)$. Then $M$ is locally near the origin biholomorphically equivalent to the A.n quadric

$$
w=\bar{z}_{1}^{2}+\cdots+\bar{z}_{n}^{2} .
$$

Proof. The complex Morse lemma (see, e.g., [Ebeling 2007, Proposition 3.15]) states that there is a local change of coordinates near the origin in just the $z$ variables such that

$$
z_{1}^{2}+\cdots+z_{n}^{2}+\bar{r}(z)
$$

is equivalent to $z_{1}^{2}+\cdots+z_{n}^{2}$.

It is not difficult to see that the normal form for mixed-holomorphic submanifolds in $\mathbb{C}^{n+1}$ of type A. $k, k<n$, is equivalent to a local normal form for a holomorphic function in $n$ variables. Therefore, for example, the submanifold $w=\bar{z}_{1}^{2}+\bar{z}_{2}^{3}$ is of type A.1 and is not equivalent to any quadric.

\section{Formal normal form for certain type C.1 submanifolds I}

In this section we prove the formal normal form in Theorem 1.3. That is, we prove that if $M \subset \mathbb{C}^{n+1}$ is defined by

$$
w=\bar{z}_{1} z_{2}+\bar{z}_{1}^{2}+r\left(z_{1}, \bar{z}_{1}, z_{2}, z_{3}, \ldots, z_{n}\right),
$$

where $r$ is $O(3)$, then $M$ is Levi-flat and formally equivalent to

$$
w=\bar{z}_{1} z_{2}+\bar{z}_{1}^{2} .
$$

That $M$ is Levi-flat follows from Proposition 6.2. 
Lemma 10.1. If $M \subset \mathbb{C}^{n+1}, n \geq 2$, is given by

$$
w=\bar{z}_{1} z_{2}+\bar{z}_{1}^{2}+r\left(z_{1}, \bar{z}_{1}, z_{2}, z_{3}, \ldots, z_{n}\right),
$$

where $r$ is an $O(3)$ formal power series, then $M$ is formally equivalent to $M_{C .1}$ given by

$$
w=\bar{z}_{1} z_{2}+\bar{z}_{1}^{2}
$$

In fact, the normalizing transformation can be of the form

$$
(z, w)=\left(z_{1}, \ldots, z_{n}, w\right) \mapsto\left(z_{1}, f(z, w), z_{3}, \ldots, z_{n}, g(z, w)\right),
$$

where $f$ and $g$ are formal power series.

Proof. Suppose that the normalization was done to degree $d-1$, then suppose that

$$
w=\bar{z}_{1} z_{2}+\bar{z}_{1}^{2}+r_{1}\left(z_{1}, \bar{z}_{1}, z_{2}, \ldots, z_{n}\right)+r_{2}\left(z_{1}, \bar{z}_{1}, z_{2}, \ldots, z_{n}\right),
$$

where $r_{1}$ is degree- $d$ homogeneous and $r_{2}$ is $O(d+1)$. Write

$$
r_{1}\left(z_{1}, \bar{z}_{1}, z_{2}, \ldots, z_{n}\right)=\sum_{j=0}^{k} \sum_{|\alpha|+j=d} c_{j, \alpha} \bar{z}_{1}^{j} z^{\alpha},
$$

where $k$ is the highest power of $\bar{z}_{1}$ in $r_{1}$, and $\alpha$ is a multiindex.

If $k$ is even, then we use the transformation that replaces $w$ with

$$
w+\sum_{|\alpha|+k=d} c_{j, \alpha} w^{k / 2} z^{\alpha}
$$

Let us look at the degree- $d$ terms in

$$
\begin{aligned}
\left(\bar{z}_{1} z_{2}+\bar{z}_{1}^{2}\right)+\sum_{|\alpha|+k=d} c_{j, \alpha}\left(\bar{z}_{1} z_{2}+\bar{z}_{1}^{2}\right)^{k / 2} z^{\alpha} & \\
& =\bar{z}_{1} z_{2}+\bar{z}_{1}^{2}+r_{1}\left(z_{1}, \bar{z}_{1}, z_{2}, \ldots, z_{n}\right) .
\end{aligned}
$$

We need not include $r_{2}$ as the terms have degree $d+1$ or higher. After canceling out the new terms on the left, we notice that the formal transformation removed all the terms in $r_{1}$ with a power $\bar{z}_{1}^{k}$ and replaced them with terms that have a smaller power of $\bar{z}_{1}$.

Next suppose that $k$ is odd. We use the transformation that replaces $z_{2}$ with

$$
z_{2}-\sum_{|\alpha|+k=d} c_{j, \alpha} w^{(k-1) / 2} z^{\alpha} .
$$


Let us look at the degree- $d$ terms in

$$
\begin{aligned}
\bar{z}_{1} z_{2}+ & \bar{z}_{1}^{2} \\
& =\bar{z}_{1}\left(z_{2}-\sum_{|\alpha|+k=d} c_{j, \alpha} w^{(k-1) / 2} z^{\alpha}\right)+\bar{z}_{1}^{2}+r_{1}\left(z_{1}, \bar{z}_{1}, z_{2}, \ldots, z_{n}\right) .
\end{aligned}
$$

Again we need not include $r_{2}$ as the terms have degree $d+1$ or higher, and we need not add the new terms to $z_{2}$ in the argument list for $r_{1}$ since all those terms would be of higher degree. Again we notice that the formal transformation removed all the terms in $r_{1}$ with a power $\bar{z}_{1}^{k}$ and replaced them with terms that have a smaller power of $\bar{z}_{1}$.

The procedure above does not change the form of the submanifold, but it lowers the degree of $\bar{z}_{1}$ by one. Since we can assume that all terms in $r_{1}$ depend on $\bar{z}_{1}$, we are finished with degree- $d$ terms after $k$ iterations of the above procedure.

\section{Convergence of normalization for certain type C.1 submanifolds}

A key point in the computation below is the following natural involution for the quadric $M_{C .1}$. Notice that the map

$$
\left(z_{1}, z_{2}, \ldots, z_{n}, w\right) \mapsto\left(-\bar{z}_{2}-z_{1}, z_{2}, \ldots, z_{n}, w\right)
$$

takes $M_{C .1}$ to itself. The involution simply replaces the $\bar{z}_{1}$ in the equation with $-z_{2}-\bar{z}_{1}$. The way this involution is defined is by noticing that the equation $w=\bar{z}_{1} z_{2}+\bar{z}_{1}^{2}$ has generically two solutions for $\bar{z}_{1}$ keeping $z_{2}$ and $w$ fixed. In the same way we could define an involution on all type C.1 submanifolds of the form $w=\bar{z}_{1} z_{2}+\bar{z}_{1}^{2}+r\left(\bar{z}_{1}, z_{2}, \ldots, z_{n}\right)$, although we will not require this construction.

We prove convergence via:

Lemma 11.1. Let $m_{1}, \ldots, m_{N}$ be positive integers. Suppose $T(z)$ is a formal power series in $z \in \mathbb{C}^{N}$. Suppose $T\left(t^{m_{1}} v_{1}, \ldots, t^{m_{N}} v_{N}\right)$ is a convergent power series in $t \in \mathbb{C}$ for all $v \in \mathbb{C}^{N}$. Then $T$ is convergent.

The proof is a standard application of the Baire category theorem and the Cauchy inequality. See [Baouendi et al. 1999, p. 153, Theorem 5.5.30], where all $m_{j}$ are one. For $m_{j}>1$ we first change variables by setting $v_{j}=w_{j}^{m_{j}}$ and apply the lemma with $m_{j}=1$.

The following lemma finishes the proof of Theorem 1.3. By absorbing any holomorphic terms into $w$, we assume that $r\left(z_{1}, 0, z_{2}, \ldots, z_{n}\right) \equiv 0$. In Lemma 10.1 we have also constructed a formal transformation that only changes the $z_{2}$ and $w$ coordinates, so it is enough to prove convergence in this case. Key points of this proof are that the right hand side of the defining equation for $M_{C .1}$ is homogeneous, and that we have a natural involution on $M_{C .1}$. 
Lemma 11.2. If $M \subset \mathbb{C}^{n+1}, n \geq 2$, is given by

$$
w=\bar{z}_{1} z_{2}+\bar{z}_{1}^{2}+r\left(z_{1}, \bar{z}_{1}, z_{2}, z_{3}, \ldots, z_{n}\right),
$$

where $r$ is $O(3)$ and convergent, and $r\left(z_{1}, 0, z_{2}, \ldots, z_{n}\right) \equiv 0$. Suppose that two formal power series $f(z, w)$ and $g(z, w)$ satisfy

$$
\begin{aligned}
& g\left(z, \bar{z}_{1} z_{2}+\bar{z}_{1}^{2}\right) \\
& \quad=\bar{z}_{1} f\left(z, \bar{z}_{1} z_{2}+\bar{z}_{1}^{2}\right)+\bar{z}_{1}^{2}+r\left(z_{1}, \bar{z}_{1}, f\left(z, \bar{z}_{1} z_{2}+\bar{z}_{1}^{2}\right), z_{3}, \ldots, z_{n}\right) .
\end{aligned}
$$

Then $f$ and $g$ are convergent.

Proof. Equation (116) is true formally, treating $z_{1}$ and $\bar{z}_{1}$ as independent variables. Notice that (116) has one equation for two unknown functions.

We now use the involution on $M_{C .1}$ to create a system that we can solve uniquely. We replace $\bar{z}_{1}$ with $-z_{2}-\bar{z}_{1}$. We leave $z_{1}$ untouched (treating as an independent variable). We obtain an identity in formal power series:

$$
\begin{aligned}
g\left(z, \bar{z}_{1} z_{2}+\bar{z}_{1}^{2}\right)=\left(-z_{2}-\right. & \left.\bar{z}_{1}\right) f\left(z, \bar{z}_{1} z_{2}+\bar{z}_{1}^{2}\right)+\left(-z_{2}-\bar{z}_{1}\right)^{2} \\
& +r\left(z_{1},\left(-z_{2}-\bar{z}_{1}\right), f\left(z, \bar{z}_{1} z_{2}+\bar{z}_{1}^{2}\right), z_{3}, \ldots, z_{n}\right) .
\end{aligned}
$$

The formal series $\xi=f\left(z, \bar{z}_{1} z_{2}+\bar{z}_{1}^{2}\right)$ and $\omega=g\left(z, \bar{z}_{1} z_{2}+\bar{z}_{1}^{2}\right)$ are solutions of the system

$$
\begin{aligned}
& \omega=\bar{z}_{1} \xi+\bar{z}_{1}^{2}+r\left(z_{1}, \bar{z}_{1}, \xi, z_{3}, \ldots, z_{n}\right), \\
& \omega=\left(-z_{2}-\bar{z}_{1}\right) \xi+\left(-z_{2}-\bar{z}_{1}\right)^{2}+r\left(z_{1},\left(-z_{2}-\bar{z}_{1}\right), \xi, z_{3}, \ldots, z_{n}\right) .
\end{aligned}
$$

We next replace $z_{j}$ with $t z_{j}$ and $\bar{z}_{1}$ with $t \bar{z}_{1}$ for $t \in \mathbb{C}$. Because $\bar{z}_{1} z_{2}+\bar{z}_{1}^{2}$ is homogeneous of degree two, we obtain that the formal series in $t$, given by $\xi(t)=$ $f\left(t z, t^{2}\left(\bar{z}_{1} z_{2}+\bar{z}_{1}^{2}\right)\right), \omega(t)=g\left(t z, t^{2}\left(\bar{z}_{1} z_{2}+\bar{z}_{1}^{2}\right)\right)$ for every $\left(z_{1}, \bar{z}_{1}, z_{2}, \ldots, z_{n}\right) \in$ $\mathbb{C}^{n+1}$, are solutions of the system

(120) $\omega=t \bar{z}_{1} \xi+t^{2} \bar{z}_{1}^{2}+r\left(t z_{1}, t \bar{z}_{1}, \xi, t z_{3}, \ldots, t z_{n}\right)$,

$$
\omega=t\left(-z_{2}-\bar{z}_{1}\right) \xi+t^{2}\left(-z_{2}-\bar{z}_{1}\right)^{2}+r\left(t z_{1}, t\left(-z_{2}-\bar{z}_{1}\right), \xi, t z_{3}, \ldots, t z_{n}\right) .
$$

We eliminate $\omega$ to obtain an equation for $\xi$,

$$
\begin{aligned}
& t\left(2 \bar{z}_{1}+z_{2}\right)\left(\xi-t z_{2}\right) \\
& \quad=r\left(t z_{1}, t\left(-z_{2}-\bar{z}_{1}\right), \xi, t z_{3}, \ldots, t z_{n}\right)-r\left(t z_{1}, t \bar{z}_{1}, \xi, t z_{3}, \ldots, t z_{n}\right)
\end{aligned}
$$

We now treat $\xi$ as a variable and we have a holomorphic (convergent) equation. The right hand side must be divisible by $t\left(2 \bar{z}_{1}+z_{2}\right)$ : It is divisible by $t$ since $r$ is divisible by $\bar{z}_{1}$. It is also divisible by $2 \bar{z}_{1}+z_{2}$ as setting $z_{2}=-2 \bar{z}_{1}$ makes the right hand side vanish. Therefore,

$$
\xi-t z_{2}=\frac{r\left(t z_{1}, t\left(-z_{2}-\bar{z}_{1}\right), \xi, t z_{3}, \ldots, t z_{n}\right)-r\left(t z_{1}, t \bar{z}_{1}, \xi, t z_{3}, \ldots, t z_{n}\right)}{t\left(2 \bar{z}_{1}+z_{2}\right)}
$$


where the right hand side is a holomorphic function (that is, a convergent power series) in $z_{1}, \bar{z}_{1}, z_{2}, \ldots, z_{n}, t, \xi$. For any fixed $z_{1}, \bar{z}_{1}, z_{2}, \ldots, z_{n}$, we solve for $\xi$ in terms of $t$ via the implicit function theorem, and we obtain that $\xi$ is a holomorphic function of $t$. The power series of $\xi$ is given by $\xi(t)=f\left(t z, t^{2}\left(\bar{z}_{1} z_{2}+\bar{z}_{1}^{2}\right)\right)$.

Let $v \in \mathbb{C}^{n+1}$ be any nonzero vector. Via a proper choice of $z_{1}, \bar{z}_{1}, z_{2}, \ldots, z_{n}$ (still treating $\bar{z}_{1}$ and $z_{1}$ as independent variables) we write $v=\left(z, \bar{z}_{1} z_{2}+\bar{z}_{1}^{2}\right)$. We apply the above argument to $\xi(t)=f\left(t v_{1}, \ldots, t v_{n}, t^{2} v_{n+1}\right)$, and $\xi(t)$ converges as a series in $t$. As we get convergence for every $v \in \mathbb{C}^{n+1}$, we obtain that $f$ converges by Lemma 11.1. Then via (120) we obtain that $g\left(t v_{1}, \ldots, t v_{n}, t^{2} v_{n+1}\right)$ converges as a series in $t$ for all $v$, and hence $g$ converges.

\section{Automorphism group of the C.1 quadric}

With the normal form achieved in previous sections, let us study the automorphism group of the C.1 quadric in this section. We will again use the mixed-holomorphic involution that is obtained from the quadric.

We study the local automorphism group at the origin. That is the set of germs at the origin of biholomorphic transformations taking $M$ to $M$ and fixing the origin.

First we look at the linear parts of automorphisms. We already know that the linear term of the last component only depends on $w$. For $M_{C .1}$ we can say more about the first two components.

Proposition 12.1. Let $(F, G)=\left(F_{1}, \ldots, F_{n}, G\right)$ be a formal invertible or biholomorphic automorphism of $M_{C .1} \subset \mathbb{C}^{n+1}$, that is the submanifold of the form

$$
w=\bar{z}_{1} z_{2}+\bar{z}_{1}^{2}
$$

Then $F_{1}(z, w)=a z_{1}+\alpha w+O(2), F_{2}(z, w)=\bar{a} z_{2}+\beta w+O(2)$, and $G(z, w)=$ $\bar{a}^{2} w+O(2)$, where $a \neq 0$.

Proof. Let $a=\left(a_{1}, \ldots, a_{n}\right)$ and $b=\left(b_{1}, \ldots, b_{n}\right)$ be such that

$$
F_{1}(z, w)=a \cdot z+\alpha w+O(2) \quad \text { and } \quad F_{2}(z, w)=b \cdot z+\beta w+O(2) .
$$

Then from Proposition 2.1 we have

$$
\left[\begin{array}{ccccc}
0 & 1 & 0 & \cdots & 0 \\
0 & 0 & 0 & \cdots & 0 \\
\vdots & \vdots & \vdots & \ddots & \vdots \\
0 & 0 & 0 & \cdots & 0
\end{array}\right]=\lambda\left[\begin{array}{lll}
a^{*} & b^{*} & \cdots
\end{array}\right]\left[\begin{array}{ccccc}
0 & 1 & 0 & \cdots & 0 \\
0 & 0 & 0 & \cdots & 0 \\
\vdots & \vdots & \vdots & \ddots & \vdots \\
0 & 0 & 0 & \cdots & 0
\end{array}\right]\left[\begin{array}{c}
a \\
b \\
\vdots
\end{array}\right] .
$$


Therefore $\lambda \bar{a}_{1} b_{2}=1$, and $\bar{a}_{j} b_{k}=0$ for all $(j, k) \neq(1,2)$. Similarly,

$$
\left[\begin{array}{ccccc}
1 & 0 & 0 & \cdots & 0 \\
0 & 0 & 0 & \cdots & 0 \\
\vdots & \vdots & \vdots & \ddots & \vdots \\
0 & 0 & 0 & \cdots & 0
\end{array}\right]=\lambda\left[\begin{array}{lll}
a^{*} & b^{*} & \cdots
\end{array}\right]\left[\begin{array}{ccccc}
1 & 0 & 0 & \cdots & 0 \\
0 & 0 & 0 & \cdots & 0 \\
\vdots & \vdots & \vdots & \ddots & \vdots \\
0 & 0 & 0 & \cdots & 0
\end{array}\right]\left[\begin{array}{c}
\bar{a} \\
\bar{b} \\
\vdots
\end{array}\right] .
$$

Therefore $\lambda \bar{a}_{1}^{2}=1$, and $\bar{a}_{j} \bar{a}_{k}=0$ for all $(j, k) \neq(1,1)$. Putting these two together we obtain that $a_{j}=0$ for all $j \neq 1$, and as $a_{1} \neq 0$ we get $b_{j}=0$ for all $j \neq 2$. As $\lambda$ is the reciprocal of the coefficient of $w$ in $G$, we are finished.

Lemma 12.2. Let $M_{C .1} \subset \mathbb{C}^{3}$ be given by

$$
w=\bar{z}_{1} z_{2}+\bar{z}_{1}^{2} .
$$

Suppose that a local biholomorphism (resp. formal automorphism) $\left(F_{1}, F_{2}, G\right)$ transforms $M_{C .1}$ into $M_{C .1}$. Then $F_{1}$ depends only on $z_{1}$, and $F_{2}$ and $G$ depend only on $z_{2}$ and $w$.

Proof. Let us define a $(1,0)$ tangent vector field on $M$ by

$$
Z=\frac{\partial}{\partial z_{2}}+\bar{z}_{1} \frac{\partial}{\partial w} .
$$

Write $F=\left(F_{1}, F_{2}, G\right) . F$ must take $Z$ into a multiple of itself when restricted to $M_{C .1}$. That is, on $M_{C .1}$ we have

$$
\begin{aligned}
& \frac{\partial F_{1}}{\partial z_{2}}+\bar{z}_{1} \frac{\partial F_{1}}{\partial w}=0, \\
& \frac{\partial F_{2}}{\partial z_{2}}+\bar{z}_{1} \frac{\partial F_{2}}{\partial w}=\lambda, \\
& \frac{\partial G}{\partial z_{2}}+\bar{z}_{1} \frac{\partial G}{\partial w}=\lambda \bar{F}_{1}(\bar{z}, \bar{w}),
\end{aligned}
$$

for some function $\lambda$. Let us take the first equation and plug in the defining equation for $M_{1}$,

$$
\frac{\partial F_{1}}{\partial z_{2}}\left(z_{1}, z_{2}, \bar{z}_{1} z_{2}+\bar{z}_{1}^{2}\right)+\bar{z}_{1} \frac{\partial F_{1}}{\partial w}\left(z_{1}, z_{2}, \bar{z}_{1} z_{2}+\bar{z}_{1}^{2}\right)=0 .
$$

This is true for all $z \in \mathbb{C}^{2}$, and so we may treat $z_{1}$ and $\bar{z}_{1}$ as independent variables.

We have an involution on $M_{C .1}$ that takes $\bar{z}_{1}$ to $-z_{2}-\bar{z}_{1}$. Therefore we also have

$$
\frac{\partial F_{1}}{\partial z_{2}}\left(z_{1}, z_{2}, \bar{z}_{1} z_{2}+\bar{z}_{1}^{2}\right)+\left(-z_{2}-\bar{z}_{1}\right) \frac{\partial F_{1}}{\partial w}\left(z_{1}, z_{2}, \bar{z}_{1} z_{2}+\bar{z}_{1}^{2}\right)=0 .
$$

This means that $\partial F_{1} / \partial w$ and therefore $\partial F_{1} / \partial z_{2}$ must be identically zero. That is, $F_{1}$ only depends on $z_{1}$. 
We have that the following must hold for all $z$ :

$$
G\left(z_{1}, z_{2}, \bar{z}_{1} z_{2}+\bar{z}_{1}^{2}\right)=\bar{F}_{1}\left(\bar{z}_{1}\right) F_{2}\left(z_{1}, z_{2}, \bar{z}_{1} z_{2}+\bar{z}_{1}^{2}\right)+\left(\bar{F}_{1}\left(\bar{z}_{1}\right)\right)^{2} .
$$

Again we treat $z_{1}$ and $\bar{z}_{1}$ as independent variables. Differentiate with respect to $z_{1}$ :

$$
\frac{\partial G}{\partial z_{1}}\left(z_{1}, z_{2}, \bar{z}_{1} z_{2}+\bar{z}_{1}^{2}\right)=\bar{F}_{1}\left(\bar{z}_{1}\right) \frac{\partial F_{2}}{\partial z_{1}}\left(z_{1}, z_{2}, \bar{z}_{1} z_{2}+\bar{z}_{1}^{2}\right) .
$$

We plug in the involution again to obtain

$$
\frac{\partial G}{\partial z_{1}}\left(z_{1}, z_{2}, \bar{z}_{1} z_{2}+\bar{z}_{1}^{2}\right)=\bar{F}_{1}\left(-z_{2}-\bar{z}_{1}\right) \frac{\partial F_{2}}{\partial z_{1}}\left(z_{1}, z_{2}, \bar{z}_{1} z_{2}+\bar{z}_{1}^{2}\right) .
$$

Therefore as $F_{1}$ is not identically zero, then as before both $\partial F_{2} / \partial z_{1}$ and $\partial G / \partial z_{1}$ must be identically zero.

Lemma 12.3. Take $M_{C .1} \subset \mathbb{C}^{3}$ given by

$$
w=\bar{z}_{1} z_{2}+\bar{z}_{1}^{2},
$$

and let $\left(F_{1}, F_{2}, G\right)$ be a local automorphism at the origin. Then $F_{1}$ uniquely determines $F_{2}$ and $G$. Furthermore, given any invertible function of one variable $F_{1}$ with $F_{1}(0)=0$, there exist unique $F_{2}$ and $G$ that complete an automorphism and they are determined by

$$
\begin{gathered}
F_{2}\left(z_{2}, \bar{z}_{1} z_{2}+\bar{z}_{1}^{2}\right)=\bar{F}_{1}\left(\bar{z}_{1}\right)+\bar{F}_{1}\left(-\bar{z}_{1}-z_{2}\right), \\
G\left(z_{2}, \bar{z}_{1} z_{2}+\bar{z}_{1}^{2}\right)=-\bar{F}_{1}\left(\bar{z}_{1}\right) \bar{F}_{1}\left(-\bar{z}_{1}-z_{2}\right) .
\end{gathered}
$$

We should note that the lemma also works formally. Given any formal $F_{1}$, there exist unique formal $F_{2}$ and $G$ satisfying the above property.

Proof. By Lemma 12.2, $F_{1}$ depends only on $z_{1}$ and $F_{2}$ and $G$ depend only on $z_{2}$ and $w$. We write the automorphism as a composition of the two mappings $\left(F_{1}\left(z_{1}\right), z_{2}, w\right)$ and $\left(z_{1}, F_{2}\left(z_{2}, w\right), G\left(z_{2}, w\right)\right)$.

We plug the transformation into the defining equation for $M_{C .1}$ and obtain

$$
G\left(z_{2}, \bar{z}_{1} z_{2}+\bar{z}_{1}^{2}\right)=\bar{F}_{1}\left(\bar{z}_{1}\right) F_{2}\left(z_{2}, \bar{z}_{1} z_{2}+\bar{z}_{1}^{2}\right)+\left(\bar{F}_{1}\left(\bar{z}_{1}\right)\right)^{2} .
$$

We use the involution $\left(z_{1}, z_{2}\right) \mapsto\left(-\bar{z}_{1}-z_{2}, z_{2}\right)$ which preserves $M_{C .1}$ and obtain a second equation

$$
G\left(z_{2}, \bar{z}_{1} z_{2}+\bar{z}_{1}^{2}\right)=\bar{F}_{1}\left(-\bar{z}_{1}-z_{2}\right) F_{2}\left(z_{2}, \bar{z}_{1} z_{2}+\bar{z}_{1}^{2}\right)+\left(\bar{F}_{1}\left(-\bar{z}_{1}-z_{2}\right)\right)^{2} .
$$

We eliminate $G$ and solve for $F_{2}$ :

$$
\begin{aligned}
F_{2}\left(z_{2}, \bar{z}_{1} z_{2}+\bar{z}_{1}^{2}\right) & =\frac{\left(\bar{F}_{1}\left(-\bar{z}_{1}-z_{2}\right)\right)^{2}-\left(\bar{F}_{1}\left(\bar{z}_{1}\right)\right)^{2}}{\bar{F}_{1}\left(\bar{z}_{1}\right)-\bar{F}_{1}\left(-\bar{z}_{1}-z_{2}\right)} \\
& =\bar{F}_{1}\left(\bar{z}_{1}\right)+\bar{F}_{1}\left(-\bar{z}_{1}-z_{2}\right) .
\end{aligned}
$$


Next we note that trivially, $F_{2}$ is unique if it exists: its difference vanishes on $M_{C .1}$.

If we suppose that $F_{1}$ is convergent, then just as before, substituting $z_{2}$ with $t z_{2}$ and $\bar{z}_{1}$ with $t \bar{z}_{1}$, we are restricting to curves $\left(t z_{2}, t^{2} w\right)$ for all $\left(z_{2}, w\right)$. The series is convergent in $t$ for every fixed $z_{2}$ and $w$. Therefore if $F_{2}$ exists and $F_{1}$ is convergent, then $F_{2}$ is convergent by Lemma 11.1 .

Now we need to show the existence of the formal solution $F_{2}$. Notice that the right hand side of (141) is invariant under the involution. It suffices to show that any power series in $\bar{z}_{1}, z_{2}$ that is invariant under the involution is a formal power series in $z_{2}$ and $\bar{z}_{1} z_{2}+\bar{z}_{1}^{2}$. Let us treat $\xi=\bar{z}_{1}$ as an independent variable. The original involution becomes a holomorphic involution in $\xi, z_{2}$ :

$$
\tau: \xi \rightarrow-\xi-z_{2}, \quad z_{2} \rightarrow z_{2}
$$

By a theorem of Noether we obtain a set of generators for the ring of invariants by applying the averaging operation $R(f)=\frac{1}{2}(f+f \circ \tau)$ to all monomials in $\xi$ and $z_{2}$ of degree two or lower. By direct calculation it is not difficult to see that $\xi$, $\xi z_{2}+\xi^{2}$ generate the ring of invariants. Therefore any invariant power series in $z_{2}$, $\xi$ is a power series in $\xi, \xi z_{2}+\xi^{2}$. This shows the existence of $F_{2}$. The existence of $G$ follows in the same way.

The equation for $G\left(z_{2}, \bar{z}_{1} z_{2}+\bar{z}_{1}^{2}\right)=-\bar{F}_{1}\left(\bar{z}_{1}\right) \bar{F}_{1}\left(-\bar{z}_{1}-z_{2}\right)$ is obtained by plugging in the equation for $F_{2}$. Its existence, uniqueness, and convergence in the case where $F_{1}$ converges, follow exactly the same as for $F_{2}$.

Theorem 12.4. If $M \subset \mathbb{C}^{n+1}, n \geq 2$ is given by

$$
w=\bar{z}_{1} z_{2}+\bar{z}_{1}^{2}
$$

and $\left(F_{1}, F_{2}, \ldots, F_{n}, G\right)$ is a local automorphism at the origin, then $F_{1}$ depends only on $z_{1}, F_{2}$ and $G$ depend only on $z_{2}$ and $w$, and $F_{1}$ completely determines $F_{2}$ and $G$ via (138). The mapping $\left(z_{1}, z_{2}, F_{3}, \ldots, F_{n}\right)$ has rank $n$ at the origin.

Furthermore, given any invertible function $F_{1}$ of one variable with $F_{1}(0)=$ 0 , and arbitrary holomorphic functions $F_{3}, \ldots, F_{n}$ such that $F_{j}(0)=0$, and $\left(z_{1}, z_{2}, F_{3}, \ldots, F_{n}\right)$ has rank $n$ at the origin, then there exist unique $F_{2}$ and $G$ so that $\left(F_{1}, \ldots, F_{n}, G\right)$ is an automorphism.

Proof. Let $\left(F_{1}, \ldots, F_{n}, G\right)$ be an automorphism. Then we have

$$
\begin{aligned}
& G\left(z_{1}, \ldots, z_{n}, w\right) \\
& \quad=\bar{F}_{1}\left(\bar{z}_{1}, \ldots, \bar{z}_{n}, \bar{w}\right) F_{2}\left(z_{1}, \ldots, z_{n}, w\right)+\left(\bar{F}_{1}\left(\bar{z}_{1}, \ldots, \bar{z}_{n}, \bar{w}\right)\right)^{2} .
\end{aligned}
$$

Proposition 12.1 says that the linear terms in $G$ only depend on $w$, the linear terms of $F_{1}$ depend only on $z_{1}$ and $w$, and the linear terms of $F_{2}$ only depend on $z_{2}$ and $w$. 
Let us embed $M_{C .1} \subset \mathbb{C}^{3}$ into $M$ via setting $z_{3}=\alpha_{3} z_{2}, \ldots, z_{n}=\alpha_{n} z_{2}$, for arbitrary $\alpha_{3}, \ldots, \alpha_{n}$. Then we obtain

$$
\begin{aligned}
& G\left(z_{1}, z_{2}, \alpha_{3} z_{2}, \ldots, \alpha_{n} z_{2}, w\right) \\
& =\bar{F}_{1}\left(\bar{z}_{1}, \bar{z}_{2}, \bar{\alpha}_{3} \bar{z}_{2}, \ldots, \bar{\alpha}_{n} \bar{z}_{2}, \bar{w}\right) F_{2}\left(z_{1}, z_{2}, \alpha_{3} z_{2}, \ldots, \alpha_{n} z_{2}, w\right) \\
& +\left(\bar{F}_{1}\left(\bar{z}_{1}, \bar{z}_{2}, \bar{\alpha}_{3} \bar{z}_{2}, \ldots, \bar{\alpha}_{n} \bar{z}_{2}, \bar{w}\right)\right)^{2} .
\end{aligned}
$$

By noting what the linear terms are, we notice that the above is the equation for an automorphism of $M_{C .1}$. Therefore by Lemma 12.2 we have

$$
\frac{\partial F_{1}}{\partial w}=0, \quad \frac{\partial F_{2}}{\partial z_{1}}=0, \quad \text { and } \quad \frac{\partial G}{\partial z_{1}}=0,
$$

as that is true for all $\alpha_{3}, \ldots, \alpha_{n}$. Plugging in the defining equation for $M_{C .1}$, we obtain an equation that holds for all $z$ and we can treat $z$ and $\bar{z}$ independently. We plug in $z=0$ to obtain

$$
\begin{aligned}
0=\bar{F}_{1}\left(\bar{z}_{1}, \bar{z}_{2}, \bar{\alpha}_{3} \bar{z}_{2}, \ldots, \bar{\alpha}_{n} \bar{z}_{2}, 0\right) F_{2}\left(0, \ldots, 0, \bar{z}_{1}^{2}\right) \\
+\left(\bar{F}_{1}\left(\bar{z}_{1}, \bar{z}_{2}, \bar{\alpha}_{3} \bar{z}_{2}, \ldots, \bar{\alpha}_{n} \bar{z}_{2}, 0\right)\right)^{2}
\end{aligned}
$$

Differentiating with respect to $\bar{\alpha}_{j}$ we obtain $\partial F_{1} / \partial z_{j}=0$, for $j=3, \ldots, n$. We set $\bar{\alpha}_{j}=0$ in the equation, differentiate with respect to $\bar{z}_{2}$ and obtain that $\partial F_{1} / \partial z_{2}=0$. In other words, $F_{1}$ is a function of $z_{1}$ only. We rewrite (145) by writing $F_{1}$ as a function of $z_{1}$ only and $F_{2}$ and $G$ as functions of $z_{2}, \ldots, z_{n}, w$, and we plug in $w=\bar{z}_{1} z_{2}+\bar{z}_{1}^{2}$ to obtain

$$
\begin{aligned}
G\left(z_{2}, \alpha_{3} z_{2}, \ldots,\right. & \left.\alpha_{n} z_{2}, \bar{z}_{1} z_{2}+\bar{z}_{1}^{2}\right) \\
& =\bar{F}_{1}\left(\bar{z}_{1}\right) F_{2}\left(z_{2}, \alpha_{3} z_{2}, \ldots, \alpha_{n} z_{2}, \bar{z}_{1} z_{2}+\bar{z}_{1}^{2}\right)+\left(\bar{F}_{1}\left(\bar{z}_{1}\right)\right)^{2} .
\end{aligned}
$$

By Lemma 12.3, we know that $F_{1}$ uniquely determines $F_{2}\left(z_{2}, \alpha_{3} z_{2}, \ldots, \alpha_{n} z_{2}, w\right)$ and $G\left(z_{2}, \alpha_{3} z_{2}, \ldots, \alpha_{n} z_{2}, w\right)$. These two functions therefore do not depend on $\alpha_{3}, \ldots, \alpha_{n}$, and in turn $F_{2}$ and $G$ do not depend on $z_{3}, \ldots, z_{n}$ as claimed. Furthermore $F_{1}$ does uniquely determine $F_{2}$ and $G$.

Finally since the mapping is a biholomorphism, and from what we know about the linear parts of $F_{1}, F_{2}$, and $G$, it is clear that $\left(z_{1}, z_{2}, F_{3}, \ldots, F_{n}\right)$ has rank $n$.

The other direction follows by applying Lemma 12.3. We start with $F_{1}$, determine $F_{2}$ and $G$ as in three dimensions. Then add $F_{3}, \ldots, F_{n}$ and the rank condition guarantees an automorphism.

\section{Normal form for certain type C.1 submanifolds II}

The goal of this section is to find the normal form for Levi-flat submanifolds $M \subset \mathbb{C}^{n+1}$ given by

$$
w=\bar{z}_{1} z_{2}+\bar{z}_{1}^{2}+\operatorname{Re} f(z),
$$


for a holomorphic $f(z)$ of order $O(3)$.

Since $f(z)$ can be absorbed into $w$ via a holomorphic transformation, the goal is really to prove:

Theorem 13.1. Let $M \subset \mathbb{C}^{n+1}$ be a real-analytic Levi-flat given by

$$
w=\bar{z}_{1} z_{2}+\bar{z}_{1}^{2}+r(\bar{z})
$$

where $r$ is $O(3)$. Then $M$ can be put into the $M_{C .1}$ normal form

$$
w=\bar{z}_{1} z_{2}+\bar{z}_{1}^{2}
$$

by a convergent normalizing transformation.

Furthermore, if $r$ is a polynomial and the coefficient of $\bar{z}_{1}^{3}$ in $r$ is zero, then there exists an invertible polynomial mapping taking $M_{C .1}$ to $M$.

In Theorem 1.3, we have already shown that a submanifold of the form

$$
w=\bar{z}_{1} z_{2}+\bar{z}_{1}^{2}+r\left(\bar{z}_{1}\right)
$$

is necessarily Levi-flat and has the normal form $M_{C .1}$. The first part of Theorem 13.1 will follow once we prove:

Lemma 13.2. If $M \subset \mathbb{C}^{n+1}$ is given by

$$
w=\bar{z}_{1} z_{2}+\bar{z}_{1}^{2}+r(\bar{z}),
$$

where $r$ is $O(3)$ and $M$ is Levi-flat, then $r$ depends only on $\bar{z}_{1}$.

Proof. First let us assume that $n=2$. For $p \in M_{\mathrm{CR}}, T_{p}^{(1,0)} M$ is one dimensional. The Levi map is the matrix

$$
L=\left[\begin{array}{lll}
0 & 1 & 0 \\
0 & 0 & 0 \\
0 & 0 & 0
\end{array}\right]
$$

applied to the $T^{(1,0)} M$ vectors. As $M$ is Levi-flat, then the Levi map has to vanish. The only vectors $v$ for which $v^{*} L v=0$, are the ones without $\partial / \partial z_{1}$ component or $\partial / \partial z_{2}$ component, that is, vectors of the form

$$
a \frac{\partial}{\partial z_{1}}+b \frac{\partial}{\partial w} \quad \text { or } \quad a \frac{\partial}{\partial z_{2}}+b \frac{\partial}{\partial w} .
$$

We apply these vectors to the defining equation and its conjugate and we obtain in the first case the equations

$$
b=0, \quad a\left(\bar{z}_{2}+2 z_{1}+\frac{\partial \bar{r}}{\partial z_{1}}\right)=0 .
$$


This cannot be satisfied identically on $M$ since this is supposed to be true for all $z$, but $a$ cannot be identically zero and the second factor in the second equation has only one nonholomorphic term, which is $\bar{z}_{2}$.

Let us try the second form and we obtain the equations

$$
b=a \bar{z}_{1}, \quad a\left(\frac{\partial \bar{r}}{\partial z_{2}}\right)=0 .
$$

Again $a$ cannot be identically zero, and hence the second factor of the second equation $\partial \bar{r} / \partial z_{2}$ must be identically zero, which is possible only if $r$ depends only on $\bar{z}_{1}$.

Finally, it is possible to pick $b=\bar{z}_{1}$ and $a=1$, to obtain a $T^{(1,0)}$ vector field

$$
\frac{\partial}{\partial z_{2}}+\bar{z}_{1} \frac{\partial}{\partial w}
$$

and therefore these submanifolds are necessarily Levi-flat.

Next suppose that $n>2$. Notice that replacing $z_{k}$ with $\lambda_{k} \xi$ for $k \geq 2$ and then fixing $\lambda_{k}$ for $k \geq 2$, we get

$$
w=\bar{z}_{1} \lambda_{2} \xi+\bar{z}_{1}^{2}+r\left(\bar{z}_{1}, \bar{\lambda}_{2} \bar{\xi}, \ldots, \bar{\lambda}_{n} \bar{\xi}\right) .
$$

By Lemma 2.2, we obtain a Levi-flat submanifold in $\left(z_{1}, \xi, w\right) \in \mathbb{C}^{3}$, and hence can apply the above reasoning to obtain that $r\left(\bar{z}_{1}, \bar{\lambda}_{2} \bar{\xi}, \ldots, \bar{\lambda}_{n} \bar{\xi}\right)$ does not depend on $\bar{\xi}$. As this was true for any $\lambda_{k}$, we have that $r$ can only depend on $\bar{z}_{1}$.

It is left to prove the claim about the polynomial normalizing transformation:

Lemma 13.3. Suppose that $M \subset \mathbb{C}^{n+1}$ is given by

$$
w=\bar{z}_{1} z_{2}+\bar{z}_{1}^{2}+r\left(\bar{z}_{1}\right),
$$

where $r$ is a polynomial that vanishes to the fourth order. Then there exists an invertible polynomial mapping taking $M_{C .1}$ to $M$.

Proof. We will take a transformation of the form

$$
\left(z_{1}, z_{2}, w\right) \mapsto\left(z_{1}, z_{2}+f\left(z_{2}, w\right), w+g\left(z_{2}, w\right)\right) .
$$

We are therefore trying to find polynomials $f$ and $g$ that satisfy

(162) $\bar{z}_{1} z_{2}+\bar{z}_{1}^{2}+g\left(z_{2}, \bar{z}_{1} z_{2}+\bar{z}_{1}^{2}\right)=\bar{z}_{1}\left(z_{2}+f\left(z_{2}, \bar{z}_{1} z_{2}+\bar{z}_{1}^{2}\right)\right)+\bar{z}_{1}^{2}+r\left(\bar{z}_{1}\right)$.

If we simplify, we obtain

$$
g\left(z_{2}, \bar{z}_{1} z_{2}+\bar{z}_{1}^{2}\right)-\bar{z}_{1} f\left(z_{2}, \bar{z}_{1} z_{2}+\bar{z}_{1}^{2}\right)=r\left(\bar{z}_{1}\right) .
$$

Consider the involution $S:\left(\bar{z}_{1}, z_{2}\right) \rightarrow\left(-\bar{z}_{1}-z_{2}, z_{2}\right)$. Its invariant polynomials $u\left(\bar{z}_{1}, z_{2}\right)$ are precisely the polynomials in $z_{2}, z_{2} \bar{z}_{1}+\bar{z}_{1}^{2}$. The polynomial $r\left(\bar{z}_{1}\right)$ can 
be uniquely written as

$$
r^{+}\left(z_{2}, \bar{z}_{1} z_{2}+\bar{z}_{1}^{2}\right)+\left(\bar{z}_{1}+\frac{1}{2} z_{2}\right) r^{-}\left(z_{2}, \bar{z}_{1} z_{2}+\bar{z}_{1}^{2}\right)
$$

in two polynomials $r^{ \pm}$. Taking $f=-r^{-}$and $g=r^{+}+\frac{1}{2} z_{2} r^{-}$, we find the desired solutions.

\section{Normal form for general type C.1 submanifolds}

In this section we show that generically a Levi-flat type C. 1 submanifold is not formally equivalent to the quadric $M_{C .1}$ submanifold. In fact, we find a formal normal form that shows infinitely many invariants. There are obviously infinitely many invariants if we do not impose the Levi-flat condition. The trick therefore is, how to impose the Levi-flat condition and still obtain a formal normal form.

Let $M \subset \mathbb{C}^{3}$ be a real-analytic Levi-flat type C.1 submanifold through the origin. We know that $M$ is an image of $\mathbb{R}^{2} \times \mathbb{C}$ under a real-analytic $C R$ map that is a diffeomorphism onto its target; see Theorem 1.2. After a linear change of coordinates we assume that the mapping is

$$
\begin{array}{r}
(x, y, \xi) \in \mathbb{R}^{2} \times \mathbb{C} \mapsto(x+i y+a(x, y, \xi), \xi+b(x, y, \xi), \\
\left.(x-i y) \xi+(x-i y)^{2}+r(x, y, \xi)\right),
\end{array}
$$

where $a, b$ are $O(2)$ and $r$ is $O(3)$. As the mapping is a CR mapping and a local diffeomorphism, then given any such $a, b$, and $r$, the image is necessarily Levi-flat at $\mathrm{CR}$ points. Therefore the set of all these mappings gives us all type C.1 Levi-flat submanifolds.

We precompose with an automorphism of $\mathbb{R}^{2} \times \mathbb{C}$ to make $b=0$. We cannot similarly remove $a$ as any automorphism must have real-valued first two components (the new $x$ and the new $y$ ), and hence those components can only depend on $x$ and $y$ but not on $\xi$. So if $a$ depends on $\xi$, we cannot remove it by precomposing.

Next we notice that we can treat $M$ as an abstract CR manifold. Suppose we have two equivalent submanifolds $M_{1}$ and $M_{2}$, with $F$ being the biholomorphic map taking $M_{1}$ to $M_{2}$. If $M_{j}$ is the image of a map $\varphi_{j}$, then note that $\varphi_{2}^{-1}$ is CR on $\left(M_{2}\right)_{\mathrm{CR}}$. Therefore, $G=\varphi_{2}^{-1} \circ F \circ \varphi_{1}$ is CR on $\left(F \circ \varphi_{1}\right)^{-1}\left(\left(M_{2}\right)_{\mathrm{CR}}\right)$, which is dense in a neighborhood of the origin of $\mathbb{R}^{2} \times \mathbb{C}$ (the CR singularity of $M_{2}$ is a thin set, and we pull it back by two real-analytic diffeomorphisms). A real-analytic diffeomorphism that is $\mathrm{CR}$ on a dense set is a CR mapping. The same argument works for the inverse of $G$, and therefore we have a CR diffeomorphism of $\mathbb{R}^{2} \times \mathbb{C}$. We conclude: 
Proposition 14.1. If $M_{j} \subset \mathbb{C}^{3}, j=1,2$ are given by the maps $\varphi_{j}$

$$
\begin{aligned}
(x, y, \xi) & \in \mathbb{R}^{2} \times \mathbb{C} \stackrel{\varphi_{j}}{\mapsto}\left(x+i y+a_{j}(x, y, \xi), \xi+b_{j}(x, y, \xi),\right. \\
& \left.(x-i y) \xi+(x-i y)^{2}+r_{j}(x, y, \xi)\right),
\end{aligned}
$$

and $M_{1}$ and $M_{2}$ are locally biholomorphically (resp. formally) equivalent at zero, then there exist local biholomorphisms (resp. formal equivalences) $F$ and $G$ at zero, with $F\left(M_{1}\right)=M_{2}, G\left(\mathbb{R}^{2} \times \mathbb{C}\right)=\mathbb{R}^{2} \times \mathbb{C}$ as germs (resp. formally) and

$$
\varphi_{2}=F \circ \varphi_{1} \circ G \text {. }
$$

In other words, the proposition states that if we find a normal form for the mapping, we find a normal form for the submanifolds. Let us prove that the proposition also works formally.

Proof. We have to prove that $G$ restricted to $\mathbb{R}^{2} \times \mathbb{C}$ is $\mathrm{CR}$, that is, $\partial G / \partial \bar{\xi}=0$. Let us consider

$$
\varphi_{2} \circ G=F \circ \varphi_{1} .
$$

The right hand side does not depend on $\bar{\xi}$ and thus the left hand side does not either. Write $G=\left(G^{1}, G^{2}, G^{3}\right)$. Let us write $b=b_{2}$ and $r=r_{2}$ for simplicity. Taking derivative of $\varphi_{2} \circ G$ with respect to $\bar{\xi}$ we get

$$
\begin{aligned}
& G_{\bar{\xi}}^{1}+i G_{\bar{\xi}}^{2}+a_{x}(G) G_{\bar{\xi}}^{1}+a_{y}(G) G_{\bar{\xi}}^{2}+a_{\xi}(G) G_{\bar{\xi}}^{3}=0 \\
& G_{\bar{\xi}}^{3}+b_{x}(G) G_{\bar{\xi}}^{1}+b_{y}(G) G_{\bar{\xi}}^{2}+b_{\xi}(G) G_{\bar{\xi}}^{3}=0 \\
& \left(G_{\bar{\xi}}^{1}-i G_{\bar{\xi}}^{2}\right) G^{3}+\left(G^{1}-i G^{2}\right) G_{\bar{\xi}}^{3}+2\left(G^{1}-i G^{2}\right)\left(G_{\bar{\xi}}^{1}-i G_{\bar{\xi}}^{2}\right) \\
& +r_{x}(G) G_{\bar{\xi}}^{1}+r_{y}(G) G_{\bar{\xi}}^{2}+r_{\xi}(G) G_{\bar{\xi}}^{3}=0 .
\end{aligned}
$$

Suppose that the homogeneous parts of $G_{\bar{\xi}}^{j}$ are zero for all degrees up to $d-1$. If we look at the degree- $d$ homogeneous parts of the first two equations above we immediately note that it must be that $G_{\bar{\xi}}^{1}+i G_{\bar{\xi}}^{2}=0$ and $G_{\bar{\xi}}^{3}=0$ in degree $d$. We then look at the degree- $(d+1)$ part of the third equation. Recall that $[\cdot]_{d}$ is the degree- $d$ part of an expression. We get

$$
\left[G_{\bar{\xi}}^{1}-i G_{\bar{\xi}}^{2}\right]_{d}\left[G^{3}+2 G^{1}-i 2 G^{2}\right]_{1}=0 .
$$

As $G$ is an automorphism we cannot have the linear terms be linearly dependent and hence $G_{\bar{\xi}}^{1}=G_{\bar{\xi}}^{2}=0$ in degree $d$. We finish by induction on $d$.

Using the proposition we can restate the result of Theorem 1.3 by parametrization:

Corollary 14.2. A real-analytic Levi-flat type C.1 submanifold $M \subset \mathbb{C}^{3}$ is biholomorphically equivalent to the quadric $M_{C .1}$ if and only if the mapping giving $M$ is 
equivalent to a mapping of the form

$$
(x, y, \xi) \in \mathbb{R}^{2} \times \mathbb{C} \mapsto\left(x+i y, \xi,(x-i y) \xi+(x-i y)^{2}+r(x, y, \xi)\right) .
$$

That is, $M$ is equivalent to $M_{C .1}$ if and only if we can get rid of the $a(x, y, \xi)$ via pre and post composing with automorphisms. The proof of the corollary follows as a submanifold that is realized by this map must be of the form $w=$ $\bar{z}_{1} z_{2}+\bar{z}_{1}^{2}+\rho\left(z_{1}, \bar{z}_{1}, z_{2}\right)$ and we apply Theorem 1.3.

We have seen that the involution $\tau$ on $M$, in particular when $M$ is the quadric, is useful to compute the automorphism group and to construct Levi-flat submanifolds of type $C$.1. We will also need to deal with power series in $z, \bar{z}, \xi$. Thus we extend $\tau$, which is originally defined on $\mathbb{C}^{2}$, as follows:

$$
\sigma(z, \bar{z}, \xi)=(z,-\bar{z}-\xi, \xi) \text {. }
$$

Here $z, \bar{z}, \xi$ are treated as independent variables. Note that $z, \xi, w=\bar{z} \xi+\bar{z}^{2}$ are invariant by $\sigma$, while $\eta=\bar{z}+\frac{1}{2} \xi$ is skew invariant by $\sigma$. A power series in $z, \bar{z}, \xi$ that is invariant by $\sigma$ is precisely a power series in $z, \xi, w$. In general, a power series $u$ in $z, \bar{z}, \xi$ admits a unique decomposition

$$
u(z, \bar{z}, \xi)=u^{+}(z, \xi, w)+\eta u^{-}(z, \xi, w) .
$$

First we introduce degree for power series $u(z, \bar{z}, \xi)$ and weights for power series $v(z, \xi, w)$. As usual we assign degree $i+j+k$ to the monomial $z^{i} \bar{z}^{j} \xi^{k}$. We assign weight $i+j+2 k$ to the monomial $z^{i} \xi^{j} w^{k}$. For simplicity, we will call them weight in both situations. Let us also set

$$
\begin{aligned}
& {[u]_{d}(z, \bar{z}, \xi)=\sum_{i+j+k=d} u_{i j k} z^{i} \bar{z}^{j} \xi^{k},} \\
& {[v]_{d}(z, \xi, w)=\sum_{i+j+2 k=d} v_{i j k} z^{i} \xi^{j} w^{k} .}
\end{aligned}
$$

Set $[u]_{i}^{j}=[u]_{i}+\cdots+[u]_{j}$ and $[v]_{i}^{j}=[v]_{i}+\cdots+[v]_{j}$ for $i \leq j$.

Theorem 14.3. Let $M$ be a real-analytic Levi-flat type C.1 submanifold in $\mathbb{C}^{3}$. There exists a formal biholomorphic map transforming $M$ into the image of

$$
\widehat{\varphi}(z, \bar{z}, \xi)=(z+A(z, \xi, w) w \eta, \xi, w)
$$

with $\eta=\bar{z}+\frac{1}{2} \xi$ and $w=\bar{z} \xi+\bar{z}^{2}$. Suppose further that $A \not \equiv 0$. Fix $i_{*}, j_{*}, k_{*}$ such that $j_{*}$ is the largest integer satisfying $A_{i_{*} j_{*} k_{*}} \neq 0$ and $i_{*}+j_{*}+2 k_{*}=s$. Then we can achieve

$$
A_{i_{*}\left(j_{*}+n\right) k_{*}}=0, \quad n=1,2, \ldots
$$


Furthermore, the power series $A$ is uniquely determined up to the transformation

$$
A(z, \xi, w) \rightarrow \bar{c}^{3} A\left(c z, \bar{c} \xi, \bar{c}^{2} w\right), \quad c \in \mathbb{C} \backslash\{0\} .
$$

In the above normal form with $A \not \equiv 0$, the group of formal biholomorphisms that preserve the normal form consists of dilations

$$
(z, \xi, w) \rightarrow\left(v z, \bar{v} \xi, \bar{v}^{2} w\right),
$$

satisfying $\bar{v}^{3} A\left(v z, \bar{v} \xi, \bar{v}^{2} w\right)=A(z, \xi, w)$.

Proof. It will be convenient to write the CR diffeomorphism $G$ of $\mathbb{R}^{2} \times \mathbb{C}$ as $\left(G_{1}, G_{2}\right)$ where $G_{1}$ is complex-valued and depends on $z, \bar{z}$, while $G_{2}$ depends on $z, \bar{z}, \xi$. Let $M$ be the image of a mapping $\varphi$ defined by

$$
(z, \bar{z}, \xi) \stackrel{\varphi}{\mapsto}\left(z+a(z, \bar{z}, \xi), \xi, \bar{z} \xi+\bar{z}^{2}+r(z, \bar{z}, \xi)\right)
$$

with $a=O(2), r=O(3)$. We want to find a formal biholomorphic map $F$ of $\mathbb{C}^{3}$ and a formal CR diffeomorphism $G$ of $\mathbb{R}^{2} \times \mathbb{C}$ such that

$$
F \hat{\varphi} G^{-1}=\varphi
$$

with $\hat{\varphi}$ in the normal form.

To simplify the computation, we will first achieve a preliminary normal form where $r=0$ and the function $a$ is skew-invariant by $\sigma$. For the preliminary normal form we will only apply $F$ and $G$ that are tangent to the identity. We will then use the general $F$ and $G$ to obtain the final normal form.

Let us assume that $F$ and $G$ are tangent to the identity. Let $M=F\left(\hat{\varphi}\left(\mathbb{R}^{2} \times \mathbb{C}\right)\right)$ where $\hat{\varphi}$ is determined by $\hat{a}, \hat{r}$. We write

$$
F=I+\left(f_{1}, f_{2}, f_{3}\right), \quad G=I+\left(g_{1}, g_{2}\right) .
$$

The $\xi$ components in $\varphi G=F \hat{\varphi}$ give us

$$
g_{2}(z, \bar{z}, \xi)=f_{2}\left(z+\widehat{a}(z, \bar{z}, \xi), \xi, \bar{z} \xi+\bar{z}^{2}+\widehat{r}(z, \bar{z}, \xi)\right) .
$$

Thus, we are allowed to define $g_{2}$ by the above identity for any choice of $f_{2}=O(2)$. Eliminating $g_{2}$ in other components of $\varphi G=F \hat{\varphi}$, we obtain

$$
\begin{aligned}
f_{1} \circ \hat{\varphi}-g_{1} & =a \circ G-\hat{a}, \\
f_{3} \circ \widehat{\varphi}-\bar{z} f_{2} \circ \hat{\varphi} & =r \circ G-\widehat{r}+2 \eta \tilde{g}_{1}+\tilde{g}_{1} f_{2} \circ \widehat{\varphi}+\tilde{g}_{1}^{2},
\end{aligned}
$$

where $\tilde{g}_{1}(z, \bar{z})=\bar{g}_{1}(\bar{z}, z)$ and

$$
(a \circ G)(z, \bar{z}, \xi):=a\left(G_{1}(z, \bar{z}), \bar{G}_{1}(\bar{z}, z), G_{2}(z, \bar{z}, \xi)\right) .
$$


Each power series $r(z, \bar{z}, \xi)$ admits a unique decomposition

$$
r(z, \bar{z}, \xi)=r^{+}(z, \xi, w)+\eta r^{-}(z, \xi, w),
$$

where both $r^{ \pm}$are invariant by $\sigma$. Note that $r(z, \bar{z}, \xi)$ is a power series in $z$, $\xi$, and $w$, if and only if it is invariant by $\sigma$, i.e., if $r^{-}=0$. We write

$$
r^{+}=w t(k) \quad \text { or } \quad w t\left(r^{+}\right) \geq k,
$$

if $r_{a b c}^{+}=0$ for $a+b+2 c<k$. Define $r^{-}=w t(k)$ analogously and write $\eta r^{-}=$ $w t(k)$ if $r^{-}=w t(k-1)$. We write $r=w t(k)$ if $\left(r^{+}, \eta r^{-}\right)=w t(k)$. Note that

$$
r=O(k) \Rightarrow r=w t(k), \quad w t(r s) \geq w t(r)+w t(s) .
$$

The power series in $z$ and $\bar{z}$ play a special role in describing normal forms. Let us define $T^{ \pm}$via

$$
u(z, \bar{z})=\left(T^{+} u\right)(z, \xi, w)+\left(T^{-} u\right)(z, \xi, w) \eta .
$$

Let $S_{k}^{+}$(resp. $S_{k}^{-}$) be spanned by monomials in $z, \bar{z}, \xi$ which have weight $k$ and are invariant (resp. skew-invariant) by $\sigma$. Then the range of $\eta T^{-}$in $S_{k}^{-}$is a linear subspace $R_{k}$. We decompose

$$
S_{k}^{-}=R_{k} \oplus\left(S_{k}^{-} \ominus R_{k}\right)
$$

The decomposition is of course not unique. We will take

$$
S_{k}^{-} \ominus R_{k}=\bigoplus_{\substack{a+b+2 c=k-1 \\ c>0}} \mathbb{C} z^{a} \xi^{b} w^{c} \eta
$$

Here we have used $\eta=\bar{z}+\frac{1}{2} \xi, \eta^{2}=w+\frac{1}{4} \xi^{2}$, and

$$
T^{+} u(z, \xi, w)=\sum_{i, j \geq 0} \sum_{0 \leq \alpha \leq j / 2} u_{i j}\left(\begin{array}{c}
j \\
2 \alpha
\end{array}\right) z^{i}\left(w+\frac{1}{4} \xi^{2}\right)^{\alpha}\left(-\frac{1}{2} \xi\right)^{j-2 \alpha}
$$

(193) $T^{-} u(z, \xi, w)$

$$
=\sum_{\substack{i \geq 0 \\
j>0}} \sum_{0 \leq \alpha<j / 2} u_{i j}\left(\begin{array}{c}
j \\
2 \alpha+1
\end{array}\right) z^{i}\left(w+\frac{1}{4} \xi^{2}\right)^{\alpha}\left(-\frac{1}{2} \xi\right)^{j-2 \alpha-1} .
$$

In particular, we have

$$
T^{-} u(z, \xi, 0)=\sum_{\substack{i \geq 0 \\ j>0}}(-1)^{j-1} u_{i j} z^{i} \xi^{j-1} .
$$


This shows that

$$
T^{-} u(z, \xi, 0)=\frac{1}{-\xi}(u(z,-\xi)-u(z, 0)) .
$$

We are ready to show that under the condition that $g_{1}(z, \bar{z})$ has no pure holomorphic terms, there exists a unique $(F, G)$ which is tangent to the identity such that $\widehat{r}=0$,

$$
\hat{a} \in \mathcal{N}:=\bigoplus \mathcal{N}_{k}, \quad \mathcal{N}_{k}:=S_{k}^{-} \ominus R_{k} .
$$

We start with terms of weight two in (183) and (184) to get

$$
\begin{aligned}
{\left[f_{1}\right]_{2}-\left[g_{1}\right]_{2} } & =[a]_{2}-\eta\left[\hat{a}^{-}\right]_{1}, \\
{\left[f_{3}\right]_{2} } & =0 .
\end{aligned}
$$

Note that $f_{j}^{-}=0$. The first identity implies that

$$
\left[f_{1}\right]_{2}-\left[T^{+} g_{1}\right]_{2}=\left[a^{+}\right]_{2}, \quad\left[T^{-} g_{1}\right]_{1}=\left[\hat{a}^{-}\right]_{1}-\left[a^{-}\right]_{1} .
$$

The first equation is solvable with kernel defined by

$$
\left[f_{1}\right]_{k}-\left[T^{+} g_{1}\right]_{k}=0,
$$

for $k=2$. This shows that $\left[g_{1}\right]_{2}$ is still arbitrary and we use it to achieve

$$
\eta\left[\hat{a}^{-}\right]_{1} \in S_{2}^{-} \ominus R_{2}=\{0\} .
$$

Then the kernel space is defined by (200) and

$$
\left[g_{1}(z, \bar{z})-g_{1}(z, 0)\right]_{k}=0
$$

with $k=2$. In particular, under the restriction

$$
\left[g_{1}(z, 0)\right]_{k}=0,
$$

for $k=2$, we have achieved $\hat{a}^{-} \in \mathcal{N}_{2}$ by unique $\left[f_{1}\right]_{2},\left[g_{1}\right]_{2},\left[f_{2}\right]_{1},\left[f_{3}\right]_{2}$. By induction, we verify that if (203) holds for all $k$, we determine uniquely $\left[f_{1}\right]_{k}$ and $\left[g_{1}\right]_{k}$ by normalizing $[\hat{a}]_{k} \in \mathcal{N}_{k}$. We then determine $\left[f_{2}\right]_{k}$ and $\left[f_{3}\right]_{k+1}$ uniquely to normalize $[\hat{r}]_{k+1}=0$. For details, let us find formulae for the solutions. We rewrite (183) as

$$
\begin{aligned}
T^{-} g_{1} & =-\left(a \circ G-\hat{a}-f_{1} \circ \hat{\varphi}\right)^{-}, \\
\left(f_{1} \circ \hat{\varphi}\right)^{+} & =(a \circ G-\hat{a})^{+}+T^{+} g_{1} .
\end{aligned}
$$

Using (194), we can solve

$$
(-1)^{j-1} g_{1, i j}=-\left((a \circ G)^{-}\right)_{i(j-1) 0}, \quad j \geq 1, i+j=k .
$$


Then we have

(207) $\left(\hat{a}^{-}\right)_{i j 0}=0$,

$i+j=k-1$;

(208) $\quad\left(\hat{a}^{-}\right)_{i j m}=\left(\left(a \circ G-f_{1} \circ \hat{\varphi}+g_{1}\right)^{-}\right)_{i j m}, \quad m \geq 1, i+j+m=k-1$.

Note that $-\left[g_{1}\right]_{k}(z,-\bar{z})=\bar{z}\left[(a \circ G-\hat{a})^{-}\right]_{k-1}(z, \bar{z}, 0)$. We obtain

$$
\left[g_{1}\right]_{k}(z, \bar{z})=\bar{z}\left[(a \circ G-\widehat{a})^{-}\right]_{k-1}(z,-\bar{z}, 0) .
$$

Having determined $\left[g_{1}\right]_{k}$, we take

$$
\left[f_{1}\right]_{k}=\left[\left(a \circ G-\hat{a}+g_{1}\right)^{+}\right]_{k} .
$$

We then solve (184) by taking

$$
\begin{gathered}
{\left[f_{2}\right]_{k}=\left[E^{-}\right]_{k}, \quad\left[f_{3}\right]_{k+1}=\left[\left(E-\frac{1}{2} \xi f_{2}\right)^{+}\right]_{k+1},} \\
E:=r \circ G-\hat{r}+2 \eta \tilde{g}_{1}+\tilde{g}_{1} f_{2} \circ \hat{\varphi}+\tilde{g}_{1}^{2} .
\end{gathered}
$$

We have achieved the preliminary normalization.

Assume now that

$$
\begin{aligned}
& \varphi(z, \bar{z}, \xi)=\left(z+a^{-}(z, \xi, w) \eta, \xi, w\right) \\
& \hat{\varphi}(z, \bar{z}, \xi)=\left(z+\hat{a}^{-}(z, \xi, w) \eta, \xi, w\right)
\end{aligned}
$$

are in the preliminary normal form, i.e.,

$$
w\left|a^{-}(z, \xi, w), \quad w\right| \hat{a}^{-}(z, \xi, w)
$$

Let us assume that

$$
a^{-}(z, \xi, w)=w t(s), \quad\left[a^{-}\right]_{s} \not \equiv 0, \quad \hat{a}^{-}(z, \xi, w)=w t(s) .
$$

We assume that $\varphi G=F \hat{\varphi}$ with

$$
\begin{aligned}
F(z, \xi, w) & =I+\left(f_{1}, f_{2}, f_{3}\right), \\
G(z, \bar{z}, \xi) & =\left(z+g_{1}(z, \bar{z}), \xi+g_{2}(z, \bar{z}, \xi)\right) .
\end{aligned}
$$

Here $f_{i}, g_{j}$ start with terms of weight and order at least two. In particular, we have

$$
\begin{array}{ll}
f_{i}=w t(N), \quad g_{i}=w t(N), & i=1,2 ; \\
f_{3}=w t\left(N^{\prime}\right), &
\end{array}
$$

Set $(P, Q, R):=\varphi G$. Using $N \geq 2, s \geq 2$, and Taylor's theorem, we obtain 
(221)

$$
\begin{array}{r}
P=z+g_{1}(z, \bar{z})+a^{-}(z, \xi, w) \eta+a^{-}(z, \xi, w)\left(\bar{g}_{1}(\bar{z}, z)+\frac{1}{2} g_{2}(z, \bar{z}, \xi)\right) \\
+\eta \nabla a^{-}(z, \xi, w)\left(g_{1}(z, \bar{z}), g_{2}(z, \bar{z}, \xi),(\xi+2 \bar{z}) \bar{g}_{1}(\bar{z}, z)+\bar{z} g_{2}(z, \bar{z}, \xi)\right) \\
+w t(s+N+1)
\end{array}
$$

(222) $Q=\xi+g_{2}(z, \bar{z}, \xi)$,

(223) $R=w+(2 \bar{z}+\xi) \bar{g}_{1}(\bar{z}, z)+\bar{z} g_{2}(z, \bar{z}, \xi)+w t(2 N)$.

We also have $(P, Q, R)=F \hat{\varphi}$. Thus

(224) $P=z+\hat{a}^{-}(z, \xi, w) \eta+f_{1}(z, \xi, w)+\partial_{z} f_{1}(z, \xi, w) \hat{a}^{-}(z, \xi, w) \eta$

$$
+w t(N+s+1)
$$

(225) $Q=\xi+f_{2}(z, \xi, w)+\partial_{z} f_{2}(z, \xi, w) \hat{a}^{-}(z, \xi, w) \eta+w t(N+s+1)$,

(226) $R=w+f_{3}(z, \xi, w)+\partial_{z} f_{3}(z, \xi, w) \hat{a}^{-}(z, \xi, w) \eta+w t\left(N^{\prime}+s+1\right)$.

We will use the above six identities for $P, Q, R$ in two ways. First we use their lower order terms to get

(227) $f_{1}(z, \xi, w)=g_{1}(z, \bar{z})+\left(a^{-}(z, \xi, w)-\hat{a}^{-}(z, \xi, w)\right) \eta+w t(N+s)$,

(228) $f_{2}(z, \xi, w)=g_{2}(z, \bar{z}, \xi)+w t(N+s)$,

(229) $f_{3}(z, \xi, w)=(2 \bar{z}+\xi) \bar{g}_{1}(\bar{z}, z)+\bar{z} g_{2}(z, \bar{z}, \xi)+w t(2 N)+w t\left(N^{\prime}+s\right)$.

Hence, we can take $N^{\prime}=N+1$. By (227) and the preliminary normalization, we first know that

$$
\widehat{a}=a+w t(N+s-1),
$$

$$
f_{1}(z, \xi, w)=b(z)+w t(N+s), \quad g_{1}(z, \bar{z})=b(z)+w t(N+s) .
$$

We compose (229) by $\sigma$ and then take the difference of the two equations to get

$$
\begin{aligned}
& f_{2}(z, \xi, w)=-\bar{b}(\bar{z})-\bar{b}(-\bar{z}-\xi)+w t(2 N-1)+w t(N+s) \\
& f_{3}(z, \xi, w)=-\bar{z} \bar{b}(-\bar{z}-\xi)+(\bar{z}+\xi) \bar{b}(\bar{z})+w t(2 N)+w t(N+s+1)
\end{aligned}
$$

Here we have used $N^{\prime}=N+1$. Let $b(z)=b_{N} z^{N}+w t(N+1)$. Therefore, we have

$$
g_{2}(z, \bar{z}, \xi)=-\bar{b}_{N}\left(\bar{z}^{N}+(-\bar{z}-\xi)^{N}\right)+w t(N+1),
$$

$$
\begin{aligned}
& \bar{g}_{1}(\bar{z}, z)+\frac{1}{2} g_{2}(z, \bar{z}, \xi)=\eta \bar{b}_{N} \sum_{\bar{z}} \bar{z}^{i}(-\bar{z}-\xi)^{N-1-i}+w t(N+1), \\
& (2 \bar{z}+\xi) \bar{g}_{1}(\bar{z}, z)+\bar{z} g_{2}(z, \bar{z}, \xi)=\bar{b}_{N}\left(\bar{z}^{N-1}+(-\bar{z}-\xi)^{N-1}\right) w+w t(N+2) .
\end{aligned}
$$

Next, we use the two formulae for $P$ and (231) to get the identity in higher weight,

$$
\hat{a}^{-}=a^{-}+g_{1}^{-}+L b_{N}+w t(N+s), \quad f_{1}-g_{1}^{+}=w t(N+s+1) .
$$


Here we have used $f_{1}^{-}=0$ and

$$
\begin{aligned}
& \quad L b_{N}(z, \xi, w):= \\
& -N b_{N} z^{N-1}\left[a^{-}\right]_{s}(z, \xi, w)-\left[a^{-}\right]_{s}(z, \xi, w) \bar{b}_{N} \sum_{i} \bar{z}^{i}(-\bar{z}-\xi)^{N-1-i} \\
& \quad+\nabla\left[a^{-}\right]_{s} \cdot\left(b_{N} z^{N},-\bar{b}_{N}\left(\bar{z}^{N}+(-\bar{z}-\xi)^{N}\right), \bar{b}_{N} w\left(\bar{z}^{N-1}+(-\bar{z}-\xi)^{N-1}\right)\right) .
\end{aligned}
$$

Recall that $w \mid a^{-}$and $w \mid \hat{a}^{-}$. We also have that $w \mid L b_{N}(z, \xi, w)$ and $L b_{N}$ is homogenous in weighted variables and of weight $N+s-1$. This shows that $\left[g_{1}^{-}(z, \xi, 0)\right]_{N+s-1}=0$. By (194), we get

$$
\left[g_{1}(z, \bar{z})\right]_{N+s}=\left[g_{1}(z, 0)\right]_{N+s}, \quad\left[\hat{a}^{-}\right]_{s+N-1}=\left[a^{-}\right]_{s+N-1}+L b_{N} .
$$

Let us make some observations. First, $L b_{N}$ depends only on $b_{N}$ and it does not depend on coefficients of $b(z)$ of degree larger than $N$. We observe that the first identity says that all coefficients of $\left[g_{1}\right]_{N+s}$ must be zero, except that the coefficient $g_{1,(N+s) 0}$ is arbitrary. On the other hand $L b_{N}$, which has weight $N+s-1$, depends only on $g_{1, N 0}$, while $N+s-1>N$. Let us assume for the moment that we have $L b_{N} \neq 0$ for all $b_{N} \neq 0$. We will then choose a suitable complement subspace $\mathcal{N}_{N+s-1}^{*}$ in the space of weighted homogenous polynomials in $z, \xi, w$ of weight $N+s-1$ for $L b_{N}$. Then $\hat{a}^{-} \in w \sum_{N>1} \mathcal{N}_{N+s-1}^{*}$ will be the required normal form. The normal form will be obtained by the following procedures: Assume that $\varphi$ is not formally equivalent to the quadratic mapping in the preliminary normalization. We first achieve the preliminary normal form by a mapping $F^{0}=I+\left(f_{1}^{0}, f_{2}^{0}, f_{3}^{0}\right)$ and $G^{0}=I+\left(g_{1}^{0}, g_{2}^{0}\right)$ which are tangent to the identity. We can make $F^{0}$ and $G^{0}$ to be unique by requiring $f_{1}^{1}(z, 0)=0$. Then $a$ is normalized such that $\hat{a}=\hat{a}^{-} \eta$ with $\left[\hat{a}^{-}\right]_{s}$ being nonzero homogenous part of the lowest weight. We may assume that $[a]_{s+1}=[\hat{a}]_{s+1}$. Inductively, we choose $f_{1, N 00}^{1}(N=2,3, \ldots)$ to achieve $\left[\hat{a}^{-}\right]_{N+s-1} \in w \mathcal{N}_{N+s-1}^{*}$. In this step for a given $N$, we determine mappings $F^{1}=I+\left(f_{1}^{1}, f_{2}^{1}, f_{3}^{1}\right)$ and $G^{1}=I+\left(g_{1}^{1}, g_{2}^{1}\right)$ by requiring that $f_{1}^{1}(z, \xi, w)$ contains only one term $\xi^{N}$, while $f_{1}^{1}, f_{2}^{1}, g_{1}^{1}, g_{2}^{1}$ have weight at most $N$ and $f_{3}^{1}$ has weight at most $N+1$. In the process, we also show that $\left[f_{1}^{1}(z, \xi, w)\right]_{2}^{N+s}$ depends only on $z$, if we do not want to impose the restriction on $f_{1}^{1}$. Moreover, the coefficient of $\xi^{N+s-1}$ of $f_{1}^{1}$ can still be arbitrarily chosen without changing the normalization achieved for $\left[\hat{a}^{-}\right]_{N+s-1}$ via $\left[f_{1}^{1}\right]_{N}$. However, by achieving $\left[\hat{a}^{-}\right]_{N+s-1} \in w \mathcal{N}_{N+s-1}^{*}$ via $F^{1}$ and $G^{1}$, we may destroy the preliminary normalization achieved via $F_{0}$ and $G_{0}$. We will then restore the preliminary normalization via $F^{2}=I+\left(f_{1}^{2}, f_{2}^{2}, f_{3}^{2}\right), G^{2}=I+\left(g_{1}^{2}, g_{2}^{2}\right)$ satisfying $g_{1}^{2}(z, 0)=0$. This amounts to determining $g_{1}^{2}=g_{1}$ and $f_{1}^{2}=f_{1}$ via (204) and (205) for which the terms of weight at most $N+s$ have been determined by (237), and then $f_{2}^{2}=f_{2}$, $f_{3}^{2}=f_{3}, g_{2}^{2}=g_{2}$ are determined by (211), (212), and (182), respectively. This allows us to repeat the procedure to achieve the normalization in any higher weight. 
We will then remove the restriction that the normalizing mappings must be tangent to the identity. This will alter the normal form only by suitable linear dilations.

Suppose that $b_{N} \neq 0$. Let us verify that

$$
L b_{N} \neq 0 .
$$

We will also identify one of nonzero coefficients to describe the normalizing condition on $\hat{a}$. We write the two invariant polynomials

$$
\begin{aligned}
\bar{z}^{N}+(-\bar{z}-\xi)^{N} & =\lambda_{N} \xi^{N}+\sum_{j<N} p_{i j k} z^{i} \xi^{j} w^{k} \\
\sum_{i} \bar{z}^{i}(-\bar{z}-\xi)^{N-1-i} & =\lambda_{N-1}^{\prime} \xi^{N-1}+\sum_{j<N-1} q_{i j k} z^{i} \xi^{j} w^{k} .
\end{aligned}
$$

If we plug in $w=\bar{z}^{2}+\bar{z} \xi$, we obtain two polynomial identities in the variables $z, \bar{z}$, and $\xi$ :

$$
\begin{aligned}
\bar{z}^{N}+(-\bar{z}-\xi)^{N} & =\lambda{ }_{N} \xi^{N}+\sum_{j<N} p_{i j k} z^{i} \xi^{j}\left(\bar{z}^{2}+\bar{z} \xi\right)^{k} \\
\sum_{i} \bar{z}^{i}(-\bar{z}-\xi)^{N-1-i} & =\lambda_{N-1}^{\prime} \xi^{N-1}+\sum_{j<N-1} q_{i j k} z^{i} \xi^{j}\left(\bar{z}^{2}+\bar{z} \xi\right)^{k} .
\end{aligned}
$$

If we set $\bar{z}=z=0$, we obtain that

$$
\lambda_{N}=\lambda_{N}^{\prime}=(-1)^{N} .
$$

Recall that $j_{*}$ is the largest integer such that $\left(a^{-}\right)_{i_{*} j_{*} k_{*}} \neq 0$ and $i_{*}+j_{*}+2 k_{*}=s$. Since $w \mid\left[a^{-}\right]_{s}$, then $k_{*}>0$. We obtain

$$
\left(L b_{N}\right)_{i_{*}\left(j_{*}+N-1\right) k_{*}}=\left(a^{-}\right)_{i_{*} j_{*} k_{*}} \bar{b}_{N}\left(-\lambda_{N-1}^{\prime}-j_{*} \lambda_{N-1}+k_{*} \lambda_{N}\right) \neq 0 .
$$

Therefore, we can achieve

$$
\left(\hat{a}^{-}\right)_{i_{*}\left(j_{*}+n\right) k_{*}}=0, \quad n=1,2, \ldots
$$

This determines uniquely all $b_{2}, b_{3}, \ldots$.

We now remove the restriction that $F$ and $G$ are tangent to the identity. Suppose that both $\varphi$ and $\hat{\varphi}$ are in the normal form. Suppose that $F \varphi=\hat{\varphi} G$. Then looking at the quadratic terms, we know that the linear parts of $F$ and $G$ must be dilations. In fact, the linear part of $F$ must be the linear automorphism of the quadric. Thus the linear parts of $F$ and $G$ have the forms

$$
G^{\prime}:(z, \xi)=(v z, \bar{v} \xi), \quad F^{\prime}(z, \xi, w)=\left(v z, \bar{v} \xi, \bar{v}^{2} w\right) .
$$

Then $\left(F^{\prime}\right)^{-1} \hat{\varphi} G^{\prime}$ is still in the normal form. Since $\left(F^{\prime}\right)^{-1} F$ is holomorphic and $\left(G^{\prime}\right)^{-1} G$ is $\mathrm{CR}$, by the uniqueness of the normalization, we know that $F^{\prime}=F$ and 
$G^{\prime}=G$. Therefore, $F$ and $G$ change the normal form $a^{-}$as follows,

$$
a^{-}(z, \xi, w)=\bar{v} \hat{a}^{-}\left(v z, \bar{v} \xi, \bar{v}^{2} w\right), \quad v \in \mathbb{C} \backslash\{0\} .
$$

When $\left[\hat{a}^{-}\right]_{s}=\left[a^{-}\right]_{s} \neq 0$, we see that $|v|=1$. Therefore, the formal automorphism group is discrete or one-dimensional.

Coffman [2006] used an analogous method of even/odd function decomposition to obtain a quadratic normal form for non Levi-flat real-analytic $m$-submanifolds in $\mathbb{C}^{n}$ with a $\mathrm{CR}$ singularity satisfying certain nondegeneracy conditions, provided $\frac{2}{3}(n+1) \leq m<n$. He was able to achieve the convergent normalization by a rapid iteration method. Using the above decomposition of invariant and skewinvariant functions of the involution $\sigma$, one might achieve a convergent solution for approximate equations when $M$ is formally equivalent to the quadric. However, when the iteration is employed, each new CR mapping $\hat{\varphi}$ might only be defined on a domain that is proportional to that of the previous $\varphi$ by a constant factor. This is significantly different from the situations of [Moser 1985; Coffman 2006; 2010], where rapid iteration methods are applicable. Therefore, even if $M$ is formally equivalent to the quadric, we do not know if they are holomorphically equivalent.

\section{Instability of Bishop-like submanifolds}

Let us now discuss stability of Levi-flat submanifolds under small perturbations that keep the submanifolds Levi-flat, in particular, we discuss which quadratic invariants are stable when moving from point to point on the submanifold. The only stable submanifolds are A. $n$ and C.1. The Bishop-like submanifolds (or even just the Bishop invariant) are not stable under perturbation, which we show by constructing examples.

Proposition 15.1. Suppose that $M \subset \mathbb{C}^{n+1}, n \geq 2$, is a connected real-analytic real codimension-two submanifold that has a nondegenerate $C R$ singular at the origin. $M$ can be written in coordinates $(z, w) \in \mathbb{C}^{n} \times \mathbb{C}$ as

$$
w=A(z, \bar{z})+B(\bar{z}, \bar{z})+O(3),
$$

for quadratic $A$ and $B$. In a neighborhood of the origin all complex tangents of $M$ are nondegenerate, while ranks of $A$ and $B$ are upper semicontinuous. Suppose that $M$ is Levi-flat (that is, $M_{\mathrm{CR}}$ is Levi-flat). The CR singular set of $M$ that is not of type $B . \frac{1}{2}$ at the origin is a real-analytic subset of $M$ of codimension at least two, while the CR singular set of $M$ that is of type $B . \frac{1}{2}$ at the origin has codimension at least one. A.n has an isolated CR singular point at the origin and so does $C .1$ in $\mathbb{C}^{3}$. Let $S_{0} \subset M$ be the set of $C R$ singular points. There is a neighborhood $U$ of the origin such that for $S=S_{0} \cap U$ we have:

(i) If $M$ is of type A. $k$ for $k \geq 2$ at the origin, then it is of type A. $j$ at each point of $S$ for some $j \geq k$. 
(ii) If $M$ is of type C.1 at the origin, then it is of type C.1 on S. If $M$ is of type C.0 at the origin, then it is of type C.0 or C.1 on S.

(iii) There exists an $M$ that is of type B. $\gamma$ at one point and of C.1 at CR singular points arbitrarily near. Similarly there exists an $M$ of type $A .1$ at $p \in M$ that is either of type C.1, or B. $\gamma$, at points arbitrarily near $p$. There also exists an $M$ of type B. $\gamma$ at every point but where $\gamma$ varies from point to point.

Proof. First we show that the rank of $A$ and the rank of $B$ are lower semicontinuous on $S_{0}$, without imposing the Levi-flatness condition. Similarly the real dimension of the range of $A(z, \bar{z})$ is lower semicontinuous on $S_{0}$. Write $M$ as

$$
w=\rho(z, \bar{z}),
$$

where $\rho$ vanishes to second order at zero. If we move to a different point of $S_{0}$ via an affine map $(z, w) \mapsto\left(Z+z_{0}, W+w_{0}\right)$. Then we have

$$
W+w_{0}=\rho\left(Z+z_{0}, \bar{Z}+\bar{z}_{0}\right) .
$$

We compute the Taylor coefficients

$$
\begin{aligned}
W=\frac{\partial \rho}{\partial z}\left(z_{0}, \bar{z}_{0}\right) & Z+\frac{\partial \rho}{\partial \bar{z}}\left(z_{0}, \bar{z}_{0}\right) \cdot \bar{Z}+Z^{*}\left[\frac{\partial^{2} \rho}{\partial z \partial \bar{z}}\left(z_{0}, \bar{z}_{0}\right)\right] Z \\
+ & \frac{1}{2} Z^{t}\left[\frac{\partial^{2} \rho}{\partial z \partial z}\left(z_{0}, \bar{z}_{0}\right)\right] Z+\frac{1}{2} Z^{*}\left[\frac{\partial^{2} \rho}{\partial \bar{z} \partial \bar{z}}\left(z_{0}, \bar{z}_{0}\right)\right] \bar{Z}+O(3) .
\end{aligned}
$$

The holomorphic terms can be absorbed into $W$. If $(\partial \rho / \partial \bar{z})\left(z_{0}, \bar{z}_{0}\right) \cdot \bar{Z}$ is nonzero, then this complex defining function has a linear term in $W$ and a linear term in $\bar{Z}$ and the submanifold is CR at this point. Therefore the set of complex tangents of $M$ is defined by

$$
\frac{\partial \rho}{\partial \bar{z}}=0,
$$

and each complex tangent point is nondegenerate. At a complex tangent point at the origin, $A$ is given by $\left[\left(\partial^{2} \rho / \partial z \partial \bar{z}\right)\left(z_{0}, \bar{z}_{0}\right)\right]$ and $B$ is given by $\frac{1}{2}\left[\left(\partial^{2} \rho / \partial \bar{z} \partial \bar{z}\right)\left(z_{0}, \bar{z}_{0}\right)\right]$. These matrices change continuously as we move along $S$. We first conclude that all CR singular points of $M$ in a neighborhood of the origin are nondegenerate. Further holomorphic transformations act on $A$ and $B$ using Proposition 2.1. Thus the ranks of $A$ and $B$ and the real dimension of the range of $A(z, \bar{z})$ are lower semicontinuous on $S_{0}$ as claimed. Furthermore as $M$ is real-analytic, the points where the rank drops lie on a real-analytic subvariety of $S_{0}$, or in other words a thin set. Let $U$ be a small enough neighborhood of the origin so that $S=S_{0} \cap U$ is connected.

Imposing the condition that $M$ is Levi-flat, we apply Theorem 1.1. By a simple computation, unless $M$ is of type B. $\frac{1}{2}$, the set of complex tangents of $M$ has codimension at least two; and A. $n$ has an isolated CR singular point and so does 
C. 1 in $\mathbb{C}^{3}$. Item (i) follows as A. $k$ are the only types where the rank of $B$ is greater than one, and the theorem says $M$ must be one of these types. For (ii), note that since $A$ is of rank one when $M$ is C. $x$ at a point, $M$ cannot be of type A. $k$ nearby. If $M$ is of type C. 1 at a point then the range of $A$ must be of real dimension two in a neighborhood, and hence on this neighborhood $M$ cannot be of type B. $\gamma$.

Examples proving (iii) are given below.

Example 15.2. Define $M$ via

$$
w=\left|z_{1}\right|^{2}+\gamma \bar{z}_{1}^{2}+\bar{z}_{1} z_{2} z_{3} .
$$

It is Levi-flat by Proposition 6.2. At the origin $M$ is of type B. $\gamma$, but at a point where $z_{1}=z_{2}=0$ and $z_{3} \neq 0$, the submanifold is CR singular and it is of type C.1.

Example 15.3. Similarly we obtain a CR singular Levi-flat $M$ via

$$
w=\bar{z}_{1}^{2}+\bar{z}_{1} z_{2} z_{3}
$$

this $M$ is A. 1 at the origin, but C. 1 at nearby CR singular points.

Example 15.4. If we define $M$ via

$$
w=\gamma \bar{z}_{1}^{2}+\left|z_{1}\right|^{2} z_{2},
$$

then $M$ is a CR singular Levi-flat type A.1 submanifold at the origin, but type B. $\gamma$ at points where $z_{1}=0$ but $z_{2} \neq 0$.

Example 15.5. The Bishop invariant can vary from point to point. Define $M$ via

$$
w=\left|z_{1}\right|^{2}+\bar{z}_{1}^{2}\left(\gamma_{1}\left(1-z_{2}\right)+\gamma_{2} z_{2}\right),
$$

where $\gamma_{1}, \gamma_{2} \geq 0$. It is not hard to see that $M$ is Levi-flat. Again it is an image of $\mathbb{C}^{2} \times \mathbb{R}^{2}$ in a similar way as above.

At the origin, the submanifold is Bishop-like with Bishop invariant $\gamma_{1}$. When $z_{1}=0$ and $z_{2}=1$, the Bishop invariant is $\gamma_{2}$. In fact when $z_{1}=0$, the Bishop invariant at that point is $\left|\gamma_{1}\left(1-z_{2}\right)+\gamma_{2} z_{2}\right|$.

Proposition 6.2 says that this submanifold possesses a real-analytic foliation extending the Levi foliation through the singular points. Proposition 6.1 says that if a foliation on $M$ extends to a (nonsingular) holomorphic foliation, then the submanifold would be a simple product of a Bishop submanifold and $\mathbb{C}$. Therefore, if $\gamma_{1} \neq \gamma_{2}$ then the Levi foliation on $M$ cannot extend to a holomorphic foliation of a neighborhood of $M$.

\section{References}

[Baouendi et al. 1999] M. S. Baouendi, P. Ebenfelt, and L. P. Rothschild, Real submanifolds in complex space and their mappings, Princeton Mathematical Series 47, Princeton University Press, 1999. MR 2000b:32066 Zbl 0944.32040 
[Bedford 1977] E. Bedford, "Holomorphic continuation of smooth functions over Levi-flat hypersurfaces”, Trans. Amer. Math. Soc. 232 (1977), 323-341. MR 58 \#1246 Zbl 0382.32009

[Bishop 1965] E. Bishop, "Differentiable manifolds in complex Euclidean space", Duke Math. J. 32 (1965), 1-21. MR 34 \#369 Zbl 0154.08501

[Brunella 2007] M. Brunella, "Singular Levi-flat hypersurfaces and codimension one foliations", Ann. Sc. Norm. Super. Pisa Cl. Sci. (5) 6:4 (2007), 661-672. MR 2009c:32065 Zbl 1214.32012 arXiv math/0701607

[Burcea 2013] V. Burcea, "A normal form for a real 2-codimensional submanifold in $\mathbb{C}^{N+1}$ near a CR singularity”, Adv. Math. 243 (2013), 262-295. MR 3062747 Zbl 1279.32024 arXiv 1110.1118

[Burns and Gong 1999] D. Burns and X. Gong, "Singular Levi-flat real analytic hypersurfaces", Amer. J. Math. 121:1 (1999), 23-53. MR 2000j:32062 Zbl 0931.32009

[Cerveau and Lins Neto 2011] D. Cerveau and A. Lins Neto, "Local Levi-flat hypersurfaces invariants by a codimension one holomorphic foliation", Amer. J. Math. 133:3 (2011), 677-716. MR 2012e:32052 Zbl 1225.32038

[Coffman 2006] A. Coffman, "Analytic stability of the CR cross-cap", Pacific J. Math. 226:2 (2006), 221-258. MR 2007j:32038 Zbl 1123.32018

[Coffman 2009] A. Coffman, "CR singularities of real fourfolds in $\mathbb{C}^{3}$ ", Illinois J. Math. 53:3 (2009), 939-981. MR 2011m:32067 Zbl 1233.32027

[Coffman 2010] A. Coffman, Unfolding CR singularities, Memoirs of the American Mathematical Society 205:962, American Mathematical Society, Providence, RI, 2010. MR 2011f:32077 Zbl 1194.32016

[Diederich and Fornaess 1978] K. Diederich and J. E. Fornaess, "Pseudoconvex domains with real-analytic boundary”, Ann. Math. (2) 107:2 (1978), 371-384. MR 57 \#16696 Zbl 0378.32014

[Dolbeault et al. 2005] P. Dolbeault, G. Tomassini, and D. Zaitsev, "On boundaries of Levi-flat hypersurfaces in $\mathbb{C}^{n}$ ", C. R. Math. Acad. Sci. Paris 341:6 (2005), 343-348. MR 2006e:32048 Zbl 1085.32019

[Dolbeault et al. 2011] P. Dolbeault, G. Tomassini, and D. Zaitsev, "Boundary problem for Levi flat graphs", Indiana Univ. Math. J. 60:1 (2011), 161-170. MR 2952414 Zbl 1244.32019

[Ebeling 2007] W. Ebeling, Functions of several complex variables and their singularities, Graduate Studies in Mathematics 83, American Mathematical Society, Providence, RI, 2007. MR 2008c:32001 Zbl 1188.32001

[Fernández-Pérez 2013] A. Fernández-Pérez, "On Levi-flat hypersurfaces with generic real singular set”, J. Geom. Anal. 23:4 (2013), 2020-2033. MR 3107688 Zbl 1277.32042

[Garrity 2000] T. Garrity, "Global structures on CR manifolds via Nash blow-ups", Michigan Math. J. 48 (2000), 281-294. MR 2001h:32058 Zbl 0995.32023

[Gong 1994a] X. Gong, "Normal forms of real surfaces under unimodular transformations near elliptic complex tangents”, Duke Math. J. 74:1 (1994), 145-157. MR 95c:32014 Zbl 0834.32003

[Gong 1994b] X. Gong, "On the convergence of normalizations of real analytic surfaces near hyperbolic complex tangents", Comment. Math. Helv. 69:4 (1994), 549-574. MR 95j:32027 Zbl 0826.32012

[Gong 2004] X. Gong, "Existence of real analytic surfaces with hyperbolic complex tangent that are formally but not holomorphically equivalent to quadrics", Indiana Univ. Math. J. 53:1 (2004), 83-95. MR 2005a:32042 Zbl 1060.32020

[Huang 1998] X. Huang, "On an $n$-manifold in $\mathbb{C}^{n}$ near an elliptic complex tangent", J. Amer. Math. Soc. 11:3 (1998), 669-692. MR 98m:32026 Zbl 0904.32016 
[Huang and Krantz 1995] X. Huang and S. G. Krantz, "On a problem of Moser", Duke Math. J. 78:1 (1995), 213-228. MR 96f:32026 Zbl 0846.32010

[Huang and Yin 2009a] X. Huang and W. Yin, "A Bishop surface with a vanishing Bishop invariant", Invent. Math. 176:3 (2009), 461-520. MR 2010f:32039 Zbl 1171.53045

[Huang and Yin 2009b] X. Huang and W. Yin, "A codimension two CR singular submanifold that is formally equivalent to a symmetric quadric", Int. Math. Res. Not. 2009:15 (2009), 2789-2828. MR 2010f:32038 Zbl 1182.32014

[Huang and Yin 2012] X. Huang and W. Yin, "Flattening of CR singular points and analyticity of local hull of holomorphy", preprint, 2012. arXiv 1210.5146

[Kenig and Webster 1982] C. E. Kenig and S. M. Webster, "The local hull of holomorphy of a surface in the space of two complex variables", Invent. Math. 67:1 (1982), 1-21. MR 84c:32014 Zbl 0489.32007

[Kenig and Webster 1984] C. E. Kenig and S. M. Webster, "On the hull of holomorphy of an $n$ manifold in $\mathbb{C}^{n} "$, Ann. Sc. Norm. Super. Pisa Cl. Sci. (4) 11:2 (1984), 261-280. MR 86d:32019 Zbl 0558.32006

[Kohn 1979] J. J. Kohn, "Subellipticity of the $\bar{\partial}$-Neumann problem on pseudo-convex domains: sufficient conditions”, Acta Math. 142:1-2 (1979), 79-122. MR 80d:32020 Zbl 0395.35069

[Lebl 2013] J. Lebl, "Singular set of a Levi-flat hypersurface is Levi-flat", Math. Ann. 355:3 (2013), 1177-1199. MR 3020158 Zbl 06149475 arXiv 1012.5993

[Lebl et al. 2014] J. Lebl, A. Minor, R. Shroff, D. Son, and Y. Zhang, "CR singular images of generic submanifolds under holomorphic maps", Ark. Mat. 52:2 (2014), 301-327. MR 3255142 arXiv 1205.5309

[Moser 1985] J. K. Moser, "Analytic surfaces in $\mathbb{C}^{2}$ and their local hull of holomorphy", Ann. Acad. Sci. Fenn. Ser. A I Math. 10 (1985), 397-410. MR 87c:32024 Zbl 0585.32007

[Moser and Webster 1983] J. K. Moser and S. M. Webster, "Normal forms for real surfaces in $\mathbb{C}^{2}$ near complex tangents and hyperbolic surface transformations", Acta Math. 150:3-4 (1983), 255-296. MR 85c:32034 Zbl 0519.32015

[Whitney 1972] H. Whitney, Complex analytic varieties, Addison-Wesley, Reading, MA, 1972. MR 52 \#8473 Zbl 0265.32008

Received March 3, 2014.

Xianghong Gong

DEPARTMENT OF MATHEMATICS

UNIVERSITY OF WISCONSIN - MADISON

MADISON, WI 53706-1388

UNITED STATES

gong@math.wisc.edu

JIŘí LEBL

DEPARTMENT OF MATHEMATICS

OKLAHOMA STATE UNIVERSITY

STILLWATER, OK 74078

UNITED STATES

lebl@math.okstate.edu 


\title{
PACIFIC JOURNAL OF MATHEMATICS
}

\author{
msp.org/pjm
}

Founded in 1951 by E. F. Beckenbach (1906-1982) and F. Wolf (1904-1989)

\section{EDITORS}

Don Blasius (Managing Editor)

Department of Mathematics

University of California

Los Angeles, CA 90095-1555

blasius@math.ucla.edu

\author{
Paul Balmer \\ Department of Mathematics \\ University of California \\ Los Angeles, CA 90095-1555 \\ balmer@math.ucla.edu \\ Robert Finn \\ Department of Mathematics \\ Stanford University \\ Stanford, CA 94305-2125 \\ finn@math.stanford.edu \\ Sorin Popa \\ Department of Mathematics \\ University of California \\ Los Angeles, CA 90095-1555 \\ popa@math.ucla.edu
}

\author{
Vyjayanthi Chari \\ Department of Mathematics \\ University of California \\ Riverside, CA 92521-0135 \\ chari@math.ucr.edu \\ Kefeng Liu \\ Department of Mathematics \\ University of California \\ Los Angeles, CA 90095-1555 \\ liu@math.ucla.edu \\ Jie Qing \\ Department of Mathematics \\ University of California \\ Santa Cruz, CA 95064 \\ qing@ cats.ucsc.edu
}

\section{PRODUCTION}

Silvio Levy, Scientific Editor, production@msp.org

\section{SUPPORTING INSTITUTIONS}

ACADEMIA SINICA, TAIPEI

CALIFORNIA INST. OF TECHNOLOGY

INST. DE MATEMÁTICA PURA E APLICADA

KEIO UNIVERSITY

MATH. SCIENCES RESEARCH INSTITUTE

NEW MEXICO STATE UNIV.

OREGON STATE UNIV.

\author{
STANFORD UNIVERSITY \\ UNIV. OF BRITISH COLUMBIA \\ UNIV. OF CALIFORNIA, BERKELEY \\ UNIV. OF CALIFORNIA, DAVIS \\ UNIV. OF CALIFORNIA, LOS ANGELES \\ UNIV. OF CALIFORNIA, RIVERSIDE \\ UNIV. OF CALIFORNIA, SAN DIEGO \\ UNIV. OF CALIF., SANTA BARBARA
}

\author{
Daryl Cooper \\ Department of Mathematics \\ University of California \\ Santa Barbara, CA 93106-3080 \\ cooper@math.ucsb.edu \\ Jiang-Hua Lu \\ Department of Mathematics \\ The University of Hong Kong \\ Pokfulam Rd., Hong Kong \\ jhlu@maths.hku.hk \\ Paul Yang \\ Department of Mathematics \\ Princeton University \\ Princeton NJ 08544-1000 \\ yang@math.princeton.edu
}

These supporting institutions contribute to the cost of publication of this Journal, but they are not owners or publishers and have no responsibility for its contents or policies.

See inside back cover or msp.org/pjm for submission instructions.

The subscription price for 2015 is US \$420/year for the electronic version, and \$570/year for print and electronic.

Subscriptions, requests for back issues and changes of subscribers address should be sent to Pacific Journal of Mathematics, P.O. Box 4163, Berkeley, CA 94704-0163, U.S.A. The Pacific Journal of Mathematics is indexed by Mathematical Reviews, Zentralblatt MATH, PASCAL CNRS Index, Referativnyi Zhurnal, Current Mathematical Publications and Web of Knowledge (Science Citation Index).

The Pacific Journal of Mathematics (ISSN 0030-8730) at the University of California, c/o Department of Mathematics, 798 Evans Hall \#3840, Berkeley, CA 94720-3840, is published twelve times a year. Periodical rate postage paid at Berkeley, CA 94704, and additional mailing offices. POSTMASTER: send address changes to Pacific Journal of Mathematics, P.O. Box 4163, Berkeley, CA 94704-0163.

PJM peer review and production are managed by EditFLOW ${ }^{\circledR}$ from Mathematical Sciences Publishers.

\section{PUBLISHED BY}

\section{mathematical sciences publishers \\ nonprofit scientific publishing}

http://msp.org/

(C) 2015 Mathematical Sciences Publishers 


\section{PACIFIC JOURNAL OF MATHEMATICS}

Volume $275 \quad$ No. $1 \quad$ May 2015

Constant-speed ramps

OSCAR M. PERDOMO

Surfaces in $\mathbb{R}_{+}^{3}$ with the same Gaussian curvature induced by the

Euclidean and hyperbolic metrics

Nilton BARRoso and PEDRO RoITMAN

Cohomology of local systems on the moduli of principally polarized abelian surfaces

DAN PETERSEN

On certain dual $q$-integral equations

Ola A. Ashour, Mourad E. H. Ismail and Zeinab S.

MANSOUR

On a conjecture of Erdôs and certain Dirichlet series

TAPAS CHATTERJEE and M. RAM MURTY

Normal forms for CR singular codimension-two Levi-flat submanifolds

XiAnGHONG GONG and JiŘí LEBL

Measurements of Riemannian two-disks and two-spheres

FLORENT BALACHEFF

Harmonic maps from $\mathbb{C}^{n}$ to Kähler manifolds

JIANMING WAN

Eigenvarieties and invariant norms

Claus M. Sorensen

The Heegaard distances cover all nonnegative integers 